\title{
Photonics for Microwave Systems and Ultra-Wideband Signal Processing*
}

\author{
W. $\mathrm{Ng}^{* *}$ \\ Department of Electrical Engineering \\ University of Southern California \\ Los Angeles, CA 90089-0271 \\ U.S.A.
}

\begin{abstract}
The advantages of using the broadband and low-loss distribution attributes of photonics to enhance the signal processing and sensing capabilities of microwave systems are well known. In this paper, we review the progress made in the topical areas of true-time-delay beamsteering, photonic-assisted analog-to-digital conversion, RF-photonic filtering and link performances. We also provide an outlook on the emerging field of integrated microwave photonics (MWP) that promise to reduce the cost of MWP subsystems and components, while providing significantly improved form-factors for system insertion.
\end{abstract}

Keywords: Microwave Photonics, Beamsteering, Photonic Analog-to-Digital Conversion, RFPhotonic Filters, Microresonators, RF-Photonic links

\footnotetext{
* Invited Paper for the Special Issue on Integrated Microwave Photonic Signal Processing

** Corresponding Author
}

Email: willieng@usc.edu

Phone: 1-213-740-5328

Fax: 213-740-8677 


\section{Introduction}

Because of its capability to accomplish wideband modulation and signal distribution via lightweight, low loss fibers that also have high electromagnetic isolation, photonics have long been viewed as a valuable technology for extending the sensing and signal processing performances of microwave systems [1-6]. Over the past two decades, the above intrinsic attributes of photonic links were augmented in performance by the introduction of a wide array of optical technologies developed initially for the telecommunication industry. To name a few, these technologies include wavelength division multiplexing (WDM), Er-doped fiber amplifiers (EDFA), fiber-based supercontinuum generation, the stable mode-locking of lasers, and dispersion control. The availability of these photonic "tools" further spurred demonstrations [716] that show the significant advantages obtained by incorporating photonics in wideband beamforming, antenna remoting, signal generation, and in signal processing functions such as filtering and analog-to-digital (A/D) conversion. In this paper, we review some of these demonstrations, and comment on the salient features of photonics that allow them to make unique contributions towards ultra-wideband sensing and signal processing. We will also provide an outlook for the future trends of RF-photonic technology development, especially with respect to system insertion and its evolution towards chip-scale integration.

This paper is organized as follows. Section 2 provides a review of photonic approaches for accomplishing the true-time-delay steering of microwave phased array antennas. Section 3 describes the progress in photonic-assisted A/D conversion. Specifically, we summarize: (i) the characterization and use of low-jitter mode-locked (ML) lasers for impulse sampling, and (ii) the use of optical dispersion for time-stretch A/D conversion. Section 4 addresses RF-photonic filtering. In this Section, we discuss the development of finite impulse response (FIR) filters as well as infinite impulse response (IIR) filters. Section 5 reports on the recent progress made in RF-photonic links. Finally, in Section 6, we summarize our discussions and provide an outlook on the future development of microwave photonics (MWP).

\section{Wideband Steering of Microwave Phased Array Antennas via Photonics}

In the 1990's, a series of pioneering work in the optical control of microwave phased array antennas $[1-2,17-20]$ were accomplished to demonstrate true-time-delay (TTD) beamsteering, 
and the insertion of ruggedized RF-photonic links in antenna systems. It is well known that TTD steering can eliminate the phenomenon of beam squint [17] that occurs in phased arrays when "phase-shifters" - as opposed to "time-shifters" - are used to form beams at distinct RF frequencies. Specifically, beam squint refers to a drift in the direction of the beam formed by the phased array when its frequency $\left(f_{m}\right)$ is changed. When normalized by the beam-half-width $\left(\theta_{1 / 2}\right)$, the beam squint $\left(\Delta \theta_{0}\right)$, is given by:

$$
\frac{\Delta \theta_{o}}{\theta_{1 / 2}}=1.13 \sin \theta_{o} \cdot \frac{L}{\lambda_{m}} \cdot \frac{\Delta f_{m}}{f_{m}}
$$

In Eq.1, $\mathrm{L}$ and $\lambda_{\mathrm{m}}$ are, respectively, the length of the phased array and the RF-wavelength of the formed beam. From Eq. 1, we see that the penalty due to beam-squint increases with: (i) $\Delta \mathrm{f}_{\mathrm{m}} / \mathrm{f}_{\mathrm{m}}$, the array's fractional bandwidth of operation, (ii) $\mathrm{L} / \lambda \mathrm{m}$, which is proportional the number of radiating elements in the array, and (iii) the beam scan angle $\theta_{\mathrm{o}}$. It was quickly recognized that if wideband fiber optic links -serving as "time-shifters" - that could deliver programmable timedelays were employed to compensate for the path difference between radiating elements during off-axis scanning, beam-squint can be eliminated over multiple microwave bands. Fig.1a shows a dual band (L- and X-band) phased array that was constructed [17] to demonstrate photonic TTD beamsteering.

a.

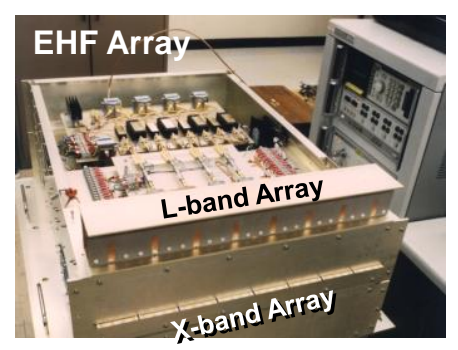

b.

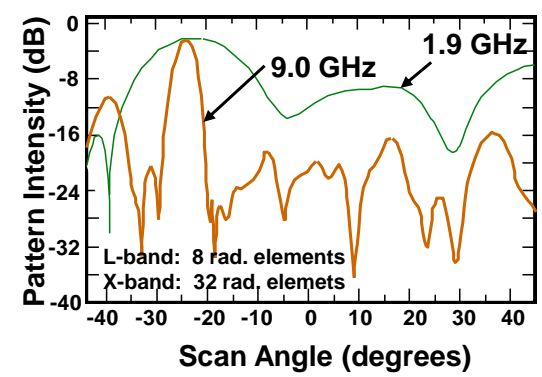

c.

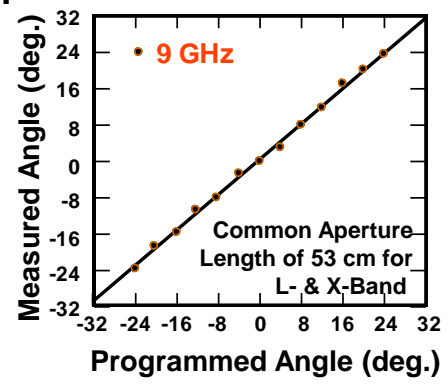

Fig.1 a. Photograph of the EHF transmit array [17] in which the L- and X-band antennas were designed to have the same array length $\mathrm{L}$ b. Squintless beams formed at $1.9 \mathrm{GHz}$ ( $\mathrm{L}$-band) and $9.0 \mathrm{GHz}$ (X-band) for $\theta_{0}=-24^{\circ}$ c. Measured angle vs programmed angle of antenna beam formed at $9 \mathrm{GHz}$

Specifically, the transmit array was designed to have radiating elements from L-band and X-band share a common array-length of $\mathrm{L} \sim 53 \mathrm{~cm}$. Four modules, each consisting of eight directly modulated fiber optic links, were used to provide TTD steering (at 3 bits of resolution) for the 
four subarrays of the antenna. In this demonstration, TTD steering was accomplished by switching optical delay-lines, hence time-delays, in accordance with the desired beam scan-angle $\theta_{\text {o. }}$ We show, in Fig.1b, the absence of beam squint as we varied the transmit frequency from 1.9 $\mathrm{GHz}$ to $9.0 \mathrm{GHz}$ for $\theta_{0}=-24^{\circ}$. As shown, the peaks of the main beams at both frequencies stayed within $0.5^{\circ}$ of each other in the measured far field pattern of the antenna. Fig. 1c shows that the measured angle of the antenna beam tracked the programmed angle from $\theta_{0}=-24^{\circ}$ to $+24^{\circ}$. Details of the optical beamforming network (BFN) design, construction and its operation are described in Ref.17 as well as in chapters of Ref. 1-2.

The above demonstration of a transmit phased array was followed by the construction of a 96-element L-band radar [19-20] that operated with 50\% instantaneous bandwidth (IBW) at Lband $(0.85-1.4 \mathrm{GHz})$. The radar, comprised of eight subarrays, was designed to form beams that support a wide scan range of $\pm 60^{\circ}$ from a conformal aperture with a $10 \mathrm{ft}$ radius of curvature (see Fig.2a). The large aperture size and wide angular scan-range for this radar imposed a differential time-delay as long as $\sim 7.75 \mathrm{nsec}$ for its BFN, which was designed to deliver 10-11 bits of resolution. We again used [19-20] the switching of optical delay-lines - formed using fibers or silica waveguides - to generate programmable time-delays for the 4-5 major bits that steer the eight subarrays. Inside the subarrays, electronic time-shifters comprised of microstrip lines were incorporated to obtain 5-6 more bits of resolution. Fig.2b and Fig.2c, show, respectively, the measured beam patterns at a transmit angle of $30^{\circ}$ and a receive angle of $60^{\circ}$ as we vary the transmit/receive frequency from $0.78 \mathrm{GHz}$ to $1.58 \mathrm{GHz}$. The absence of beam squint was evident from the measured beam patterns.

a.

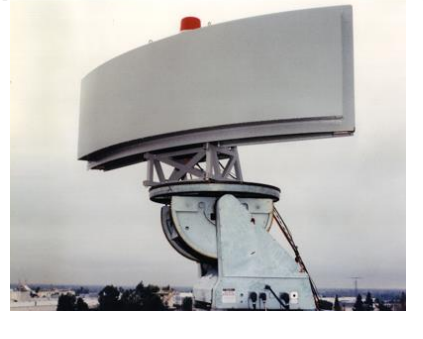

b.

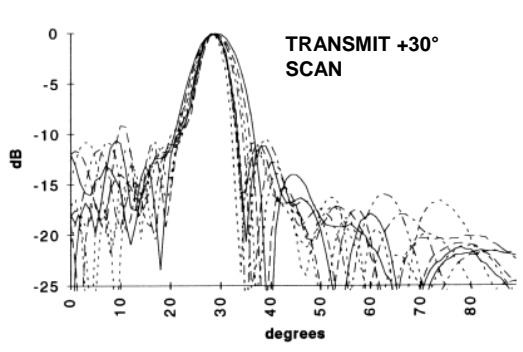

c.

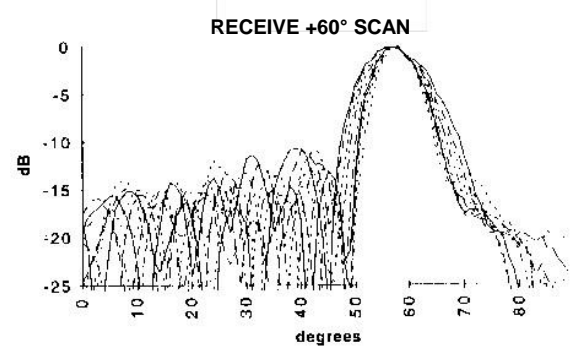

Fig.2 a. Photograph of the L-band conformal radar [19-20] with a $10 \mathrm{ft}$ radius of curvature b. Transmit beam pattern obtained for $\mathrm{a}+30^{\circ}$ scan $\mathrm{c}$. Receive beam pattern obtained for a $-60^{\circ}$ scan 
The demonstration of this L-band radar also marked the first insertion of integrated waveguide (WG) delay-lines in a phased array antenna. Specifically, the central column of the eight subarrays was steered by a set of silica WG delay-lines programmed to deliver stepped differential time-delays $\left(\Delta \mathrm{t}_{\mathrm{d}}\right)$ that ranged from $\Delta \mathrm{t}_{\mathrm{d}}=\Delta \mathrm{t}$ to $15 \Delta \mathrm{t}$, where $\Delta \mathrm{t}$ was $0.25 \mathrm{nsec}$. While the propagation loss of WGs integrated on chip was $\sim 2 \mathrm{~dB} /$ meter at the time, significant progress had since been made in the fabrication of dielectric WGs to achieve low losses of $\sim 0.1$ $0.3 \mathrm{~dB} /$ meter [21-22] near $\lambda \sim 1550 \mathrm{~nm}$. Integrated WG delay-loops (see Fig. 3), $10 \mathrm{~m}$ in length (providing a time-delay $t_{d}$ of $50 \mathrm{nsec}$ ), had also been fabricated on Si-substrates [23]. For a relative refractive index difference of $1.5 \%$, the minimum bend radius-of-curvature $r$ (see Fig.3) of these WGs is $\sim 2 \mathrm{~mm}$ [24]. These advances will allow us to construct a low loss, chip-scale BFN formed with integrated WGs that have $\Delta \mathrm{t}_{\mathrm{d}}$ defined precisely (to $<1 \mathrm{psec}$ of accuracy) by photolithography.

With the advent of WDM technologies, a variety of TTD schemes that utilize optical wavelengths and dispersion elements to control time-delays were demonstrated. For example, in Ref. 18, a fiber prism feeding an array of photodetectors (PDs) located across an antenna aperture was constructed by connecting varying lengths of highly dispersive (HD) and nondispersive fibers. After propagation through the prism, an incident optical wavelength $\lambda$ lower (higher) than a center-wavelength designed to deliver a boresight-beam will incur longer (shorter) relative time-delays across the outputs of the fiber prism. In this manner, a beam controlled by $\lambda$ can be formed to scan over $\pm \theta_{0}$. With the development of chirped fiber gratings (CFGs) that have dispersion coefficients as high as $-2000 \mathrm{ps} / \mathrm{nm}$ (see their application for timestretching in Section 3), CFGs (with additional circulators) could also be employed in place of HD fibers to control dispersion in an optical prism type BFN [25-26].

Fig. 3 shows a wavelength-switched BFN architecture that can be used to replace the "laserswitching" scheme described in Ref. 17. In the wavelength switching scheme, all the passive components can be integrated on a Si substrate. Conceptually, the approach shown in Fig.3 is similar to the recirculating AWG described in [27]. In practice, it might offer more flexibility in accomplishing channel-uniformity, lower channel-crosstalk, and a low-loss WG-loop layout when there are large channel $(\mathrm{Ch})$ counts. 


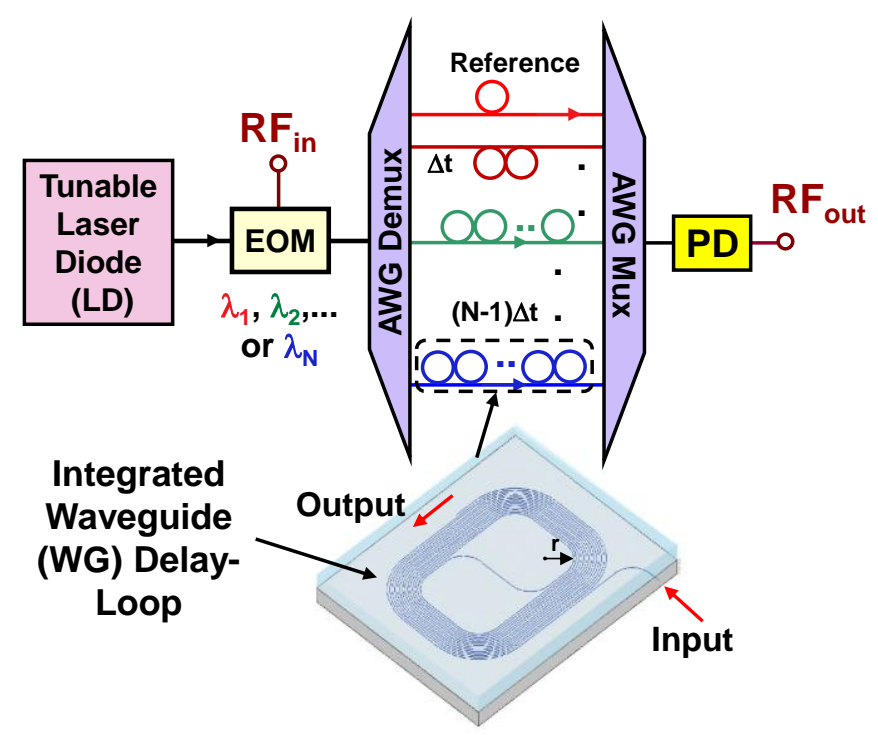

Fig.3 The generation of stepped time-delays from an integrated chip comprised of WG delay-loops and AWG mux/demux via the switching of optical wavelengths $\lambda_{1} . . \lambda_{N}$. In the figure, PD denotes photodetector.

As shown, a tunable laser diode (LD) is used to generate the optical carrier $\left(\lambda_{1}, \lambda_{2} .\right.$. or $\left.\lambda_{\mathrm{N}}\right)$ incident on an electro-optic modulator (EOM). The RF-modulated output of the EOM is then fed to an array-waveguide grating (AWG) demultiplexer (demux). Because these AWG demux or multiplexers (mux) operate as an optical phased array (via interference of Phasar-arm outputs), their on-chip optical insertion loss, e.g., for 1:N $\lambda$-demultiplexing, can be $<3 \mathrm{~dB}$ for each Ch, even for N > 250 [28]. Each of the AWG output can then directed to a WG delay-loop (see Fig.3) designed to provide stepped time-delays $\Delta t_{\text {d. }}$ Finally, the outputs of the WG delayloops are multiplexed together with a second AWG mux. By switching the lasing wavelength $(\lambda)$ of the tunable $\mathrm{LD}$, one can then vary the stepped time-delays $\left(\Delta \mathrm{t}_{\mathrm{d}}\right)$ directed at the photodetector (PD). Tunable LD technology developed to date will cover spectral widths that are $>40 \mathrm{~nm}$ [29]. Combined with high resolution (e.g., a Ch-separation of $50 \mathrm{GHz}$ ) AWG mux/demux that are already developed, a large number time-delay steps (for achieving fine beam-resolution) can be generated with the scheme depicted in Fig.3. In addition, the $\lambda$-tuning can be accomplished in switching times that are $<5 \mathrm{nsec}$ [30], making the scheme very agile for beam reconfiguration. Finally, our AWG mux/demux, as well as the WG delay-loops can all be integrated on a planar lightwave circuit (PLC) platform [28]. With the above monolithic integration, we expect the 
operational phase-stability of these optical BFNs to be very robust with respect to environmental perturbations.

The rapid development of Si-photonics and optical ring resonators (ORR) based on TriPleX ${ }^{\mathrm{TM}}$ [11], a silicon nitride/silicon dioxide WG technology, have stimulated much interest [31-35] to demonstrate integrated BFN formed by tailoring the group-delays $\left(\tau_{\mathrm{g}}\right)$ of cascaded ORRs. The bend radius of curvature for these TriPleX ${ }^{\mathrm{TM}}$ WGs is less than $70 \mu \mathrm{m}$, making them an attractive technology for monolithic integration. In these BFNs, the time-delay $\tau_{\mathrm{g}}$ is given by the derivative $-\partial \phi(\omega) / \partial \omega$ of the optical phase-response $(\phi(\omega))$ in an ORR, where $\omega$ is the optical frequency. Multiple ORRs are cascaded to increase the available delay-bandwidth, while keeping the delay-ripples below an acceptable value (see Fig.4).

a.

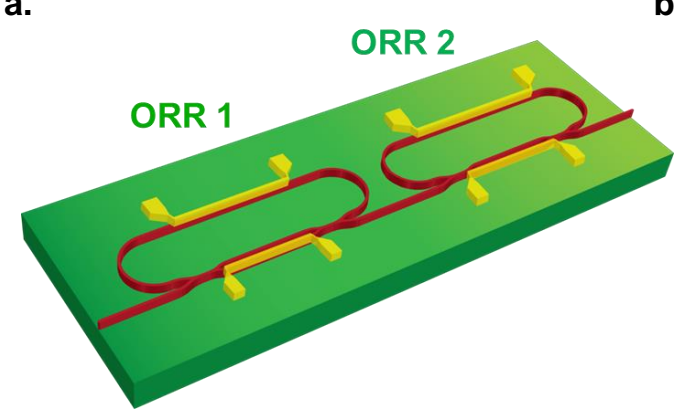

b.

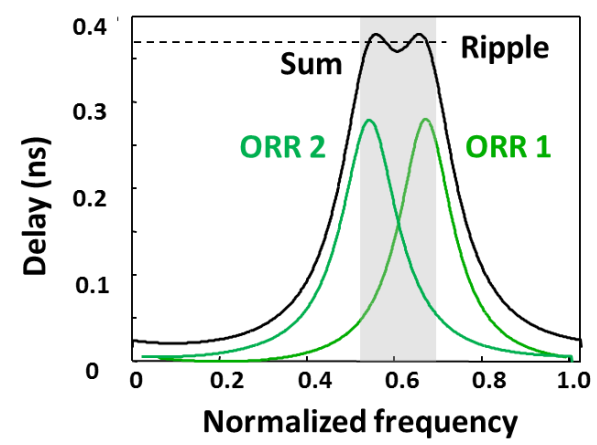

Fig.4 a. Cascaded optical ring resonator (ORR) integrated on a chip to accomplish tunable delay over a wider optical bandwidth b. Time-delay obtained for cascaded ORR 1 and ORR 2, showing the tradeoff between bandwidth and ripple [35]

System wise, the above tradeoff amounts to obtaining sufficiently large time-delays and optical bandpasses, while staying within acceptable phase-errors during beamforming. Using these cascaded ORRs, continuous time-delays for beamforming have been demonstrated with use of a single wavelength, multi-wavelengths (for hardware compression), as well as for multiple beam formation [35].

In summary, a variety of TTD beamforming architectures have been demonstrated over the past two decades. The intrinsically small size of optical devices (derived from their much smaller wavelength compared with microwaves), accompanied by the advancement of WDM and integrated photonic technologies, should enable us to develop rapidly reconfigurable, chip- 
scale BFNs that can steer large phased arrays. To make a significant impact on the microwave antenna community, the photonic links [3, 12, 36-37] used in these BFNs must exhibit gain and noise figure, as well as spur free dynamic range (SFDR on receive) that meet their expectations (see also Section 5). Eq. 1 shows that the performance gains achieved via TTD steering are in synergy with recent goals to achieve multiband scanning over wide angles and with high resolution. These goals should further incentivize the development of integrated optical BFN technologies, so that wideband photonic beamforming can be accomplished with attractive formfactors for system insertion.

\section{Photonic-Assisted Analog-to-Digital Conversion}

The interest in developing photonic-assisted A/D conversion was partially motivated by the potential to clock analog-to-digital converters (ADCs) with the low-jitter pulses generated by mode-locked (ML) lasers, so that a large effective-number-of-bits (ENOB) can be achieved at high sampling rates $\left(f_{s}\right)$, e.g., at $f_{s}=10 \mathrm{Gs} / \mathrm{s}$. The seminal surveys of Walden [38-39] indicated that "aperture-uncertainty" caused the attained ENOB of ADCs to drop at the rate of -3.3

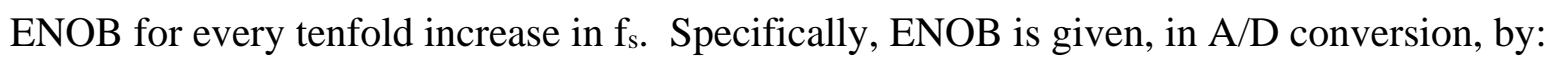

$$
E N O B=\frac{S N D R-1.76}{6.02}
$$

where SNDR is the signal-to-noise-and-distortion ratio [38]. Consequently, early efforts in photonic ADC involved the detailed characterizations [40-43] of the phase-noise and timingjitter of ML pulse trains. Fig.5a shows an experimental arrangement [41] for measuring the absolute phase $(\phi)$ noise of a fiber-laser actively mode-locked at $\mathrm{f}_{\mathrm{ML}}=10.24 \mathrm{GHz}$. The reference oscillator in this work was a sapphire-loaded cavity resonator (SLCR), considered as one of the most stable RF-oscillators available to date [41]. To obtain a baseband-signal for $\phi$-noise measurement, we first downconverted the $10.24 \mathrm{GHz}$ harmonic of the photodetected pulse train with a second SLCR oscillator at $9.0 \mathrm{GHz}$. Using a second stage of downconversion, we obtained an IF signal (at $40 \mathrm{MHz}$ ) that was subsequently fed to the R-input of our $\phi$-noise test set. Inside the $\phi$-noise test set, the $40 \mathrm{MHz}$ signal was mixed with a reference at the same frequency, but locked at quadrature. The single sideband $\phi$-noise L(f) thus measured was plotted as curve 2 in Fig.5b. As shown, the L(f) of the ML fiber laser tracked closely that of the 
10.24GHz SLCR oscillator measured (curve 1) via use of the same downconversion arrangement.

a.

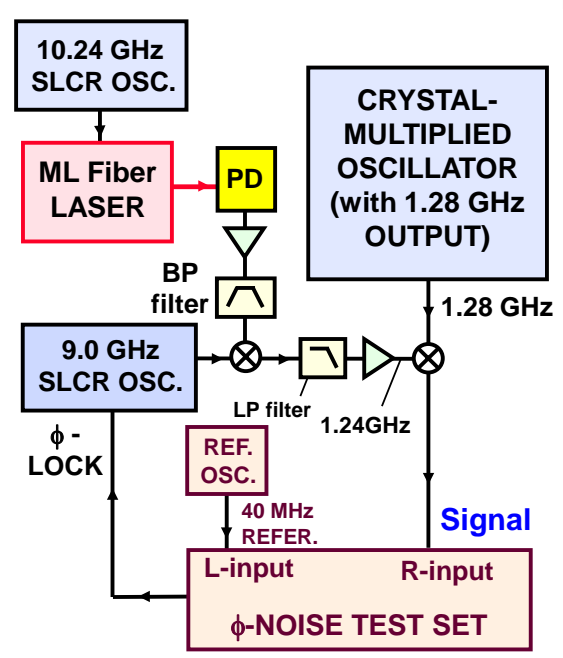

b.

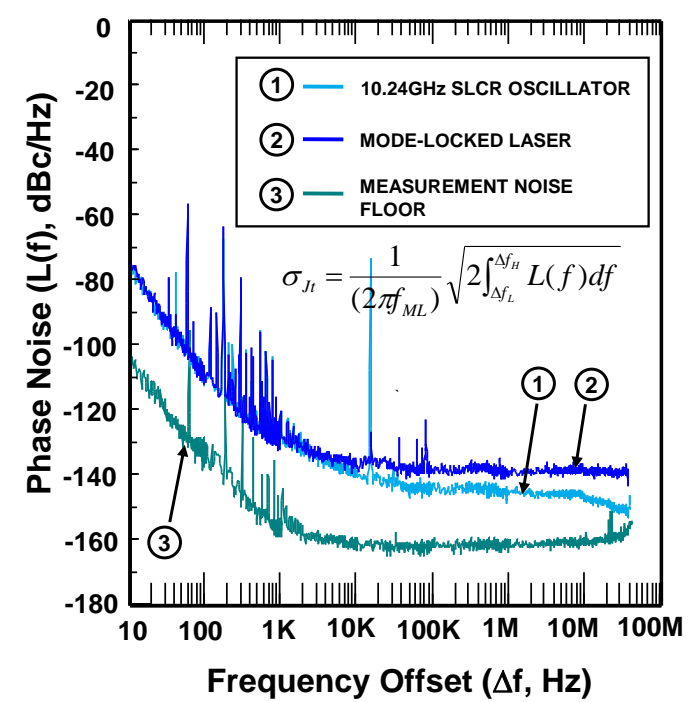

Fig.5 a. Experimental setup for measuring the absolute $\phi$-noise of a fiber laser ML by a SLCR oscillator at the frequency of $f_{M L}=10.24 \mathrm{GHz}$ b. Measured single sideband $\phi$-noise $\mathrm{L}(\mathrm{f})$ of SLCR oscillator and the ML fiber laser.

When we integrated L(f) (see equation for the absolute timing-jitter $\sigma_{\mathrm{Jt}}$ in Fig.5b) over the full offset-frequency ( $\Delta \mathrm{f}$ ) range (i.e., $\Delta \mathrm{f}=10 \mathrm{~Hz}-40 \mathrm{MHz}$ ) of measurement, we obtained an absolute timing-jitter $\sigma_{\mathrm{Jt}}$ of $16.4 \mathrm{fsec}$ for the ML laser. Integrating over an extrapolated $\Delta \mathrm{f}$-range of $\Delta \mathrm{f}=$ $10 \mathrm{~Hz}$ to $5.12 \mathrm{GHz}$, the Nyquist frequency for $\mathrm{f}_{\mathrm{ML}}=10.24 \mathrm{GHz}$, we obtained a $\sigma_{\mathrm{Jt}}$ of $70 \mathrm{fsec}$, showing that the low timing-jitter of the ML laser can support a Nyquist sampling accuracy of $\sim 8-9$ bits [40]. Finally, we take note that photonic-assisted A/D conversion had also been accomplished with short optical pulse-trains formed by compressing chirped pulses derived from a CW laser cascaded with phase- and amplitude-modulators [44].

\subsection{Photonic A/D Conversion with InP-HBT Optoelectronic Integrated Circuits (OEIC)}

One of the early demonstrations that used the "impulse-sampling" provided by low-jitter ML pulses was illustrated in Fig.6. In this work [45], the electronic quantizer was an InP heterojunction bipolar transistor (HBT) $\Delta-\Sigma$ ADC that had integrated photodiodes formed from the base-collector junction of the InP HBTs (see Fig. 7). A $11.98 \mathrm{GHz}$ analog-input fed to a 
$\mathrm{LiNbO}_{3}$ Mach Zehnder (MZ) EOM was subsampled by the $10.24 \mathrm{GHz}$ ML pulses to an IF frequency of $1.74 \mathrm{GHz}$, where a notch (centered at $\mathrm{f}_{\text {notch }}=1.76 \mathrm{GHz}$ ) for the quantization noise was formed by the $\Delta \Sigma$ feedback loop (see inset of Fig.8).

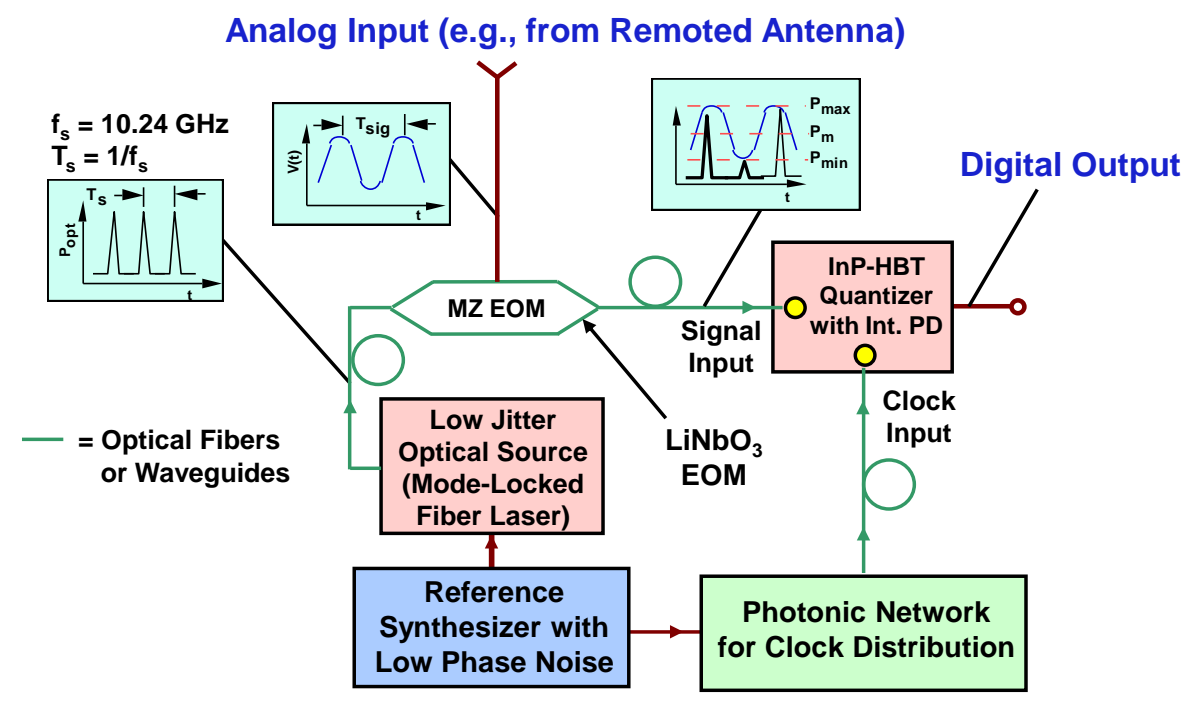

Fig.6 Photonic A/D conversion using low jitter ML pulses for impulse sampling and InP-HBT $\Delta \Sigma$ ADC for quantization. The analog-input was impressed as an envelope on the pulse train via use of an EOM. The reference synthesizer used in Ref. 45 was a SLCR 10.24 GHz oscillator.

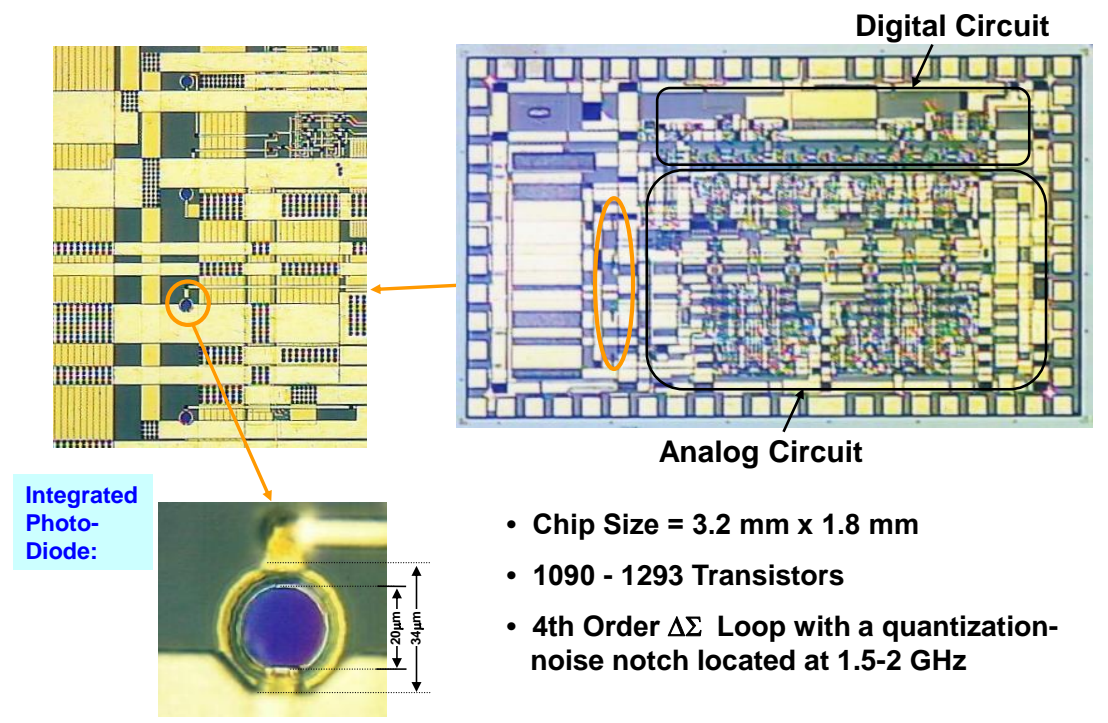

Fig.7 InP-HBT $\Delta \Sigma$ quantizer optoelectronic integrated circuit (OEIC) with integrated photodetectors, analog circuit (analog loop-filter and integrator), and a comparator (1-bit quantizer) [45] 
Integrating the L(f) in Fig.5b over an offset frequency range of $\Delta \mathrm{f}=10 \mathrm{~Hz}$ to $\Delta \mathrm{f}=1 \mathrm{MHz}$, we obtained a timing-jitter of only $8.28 \mathrm{fsec}$, which indicated that a sampling accuracy of $\sim 11.9$ bits could be achieved. Fig.8 shows that we measured a signal-to-noise ratio (SNR) of $\sim 60 \mathrm{~dB}$ for the downconverted signal at $1.74 \mathrm{GHz}$, over a noise bandwidth of $1 \mathrm{MHz}$ [45]. Using Eq. 2, we estimated an ENOB of 10 (1 MHz noise bandwidth) for the above direct sampling of a 11.98GHz analog-input.

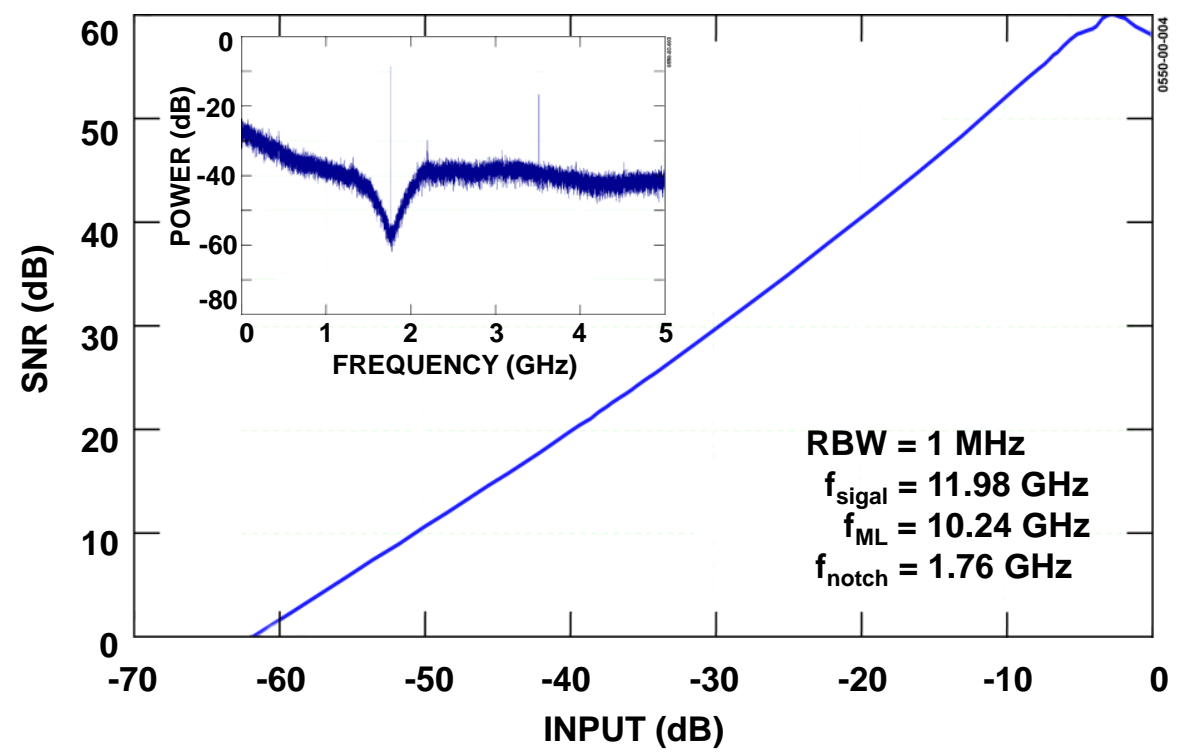

Fig.8 Measured SNR vs the relative input power (at $f_{i n}=11.98 \mathrm{GHz}$ ) to the EOM. The inset shows the 1.74GHz downconverted signal located at the quantization-notch of the InP-HBT $\Delta \Sigma$ ADC

As shown in Fig.6, the optical distribution of ML pulses and clock signals via fibers enabled us to remote the antenna, EOM and InP-HBT OEIC from the SLCR oscillator and the ML laser. In particular, the InP-HBT OEIC would have to be co-located with the SLCR oscillator to receive its $10.24 \mathrm{GHz}$ clock-signal. In the context of LO-distribution, we had also demonstrated that extremely stable CW clock-signals can be delivered from an oscillator to multiple locations with use of RF-photonic links. The residual $\phi$-noise measured for these links was lower than -140 $\mathrm{dBc} / \mathrm{Hz}$ at $\Delta \mathrm{f}=10 \mathrm{kHz}[46]$.

\subsection{Time-Stretch Photonic Analog-to-Digital Conversion}

A second approach that uses photonics to help accomplish a large ENOB at high input-signal frequencies is "time-stretching" [47]. In this approach (see Fig. 9), the stable pulse-train 
generated by a ML supercontinuum (SC) source is first chirped by a dispersion element (with dispersion coefficient $\mathrm{D}_{1}$ ), such as a length of dispersion compensating fiber (DCF). The analoginput $V_{\text {in }}(t)$ is then impressed on the chirped pulses as an envelope via use of an EOM. Because the pulses incident on the EOM are chirped, the time-variation of $\mathrm{V}_{\text {in }}(\mathrm{t})$ becomes mapped to optical wavelengths.

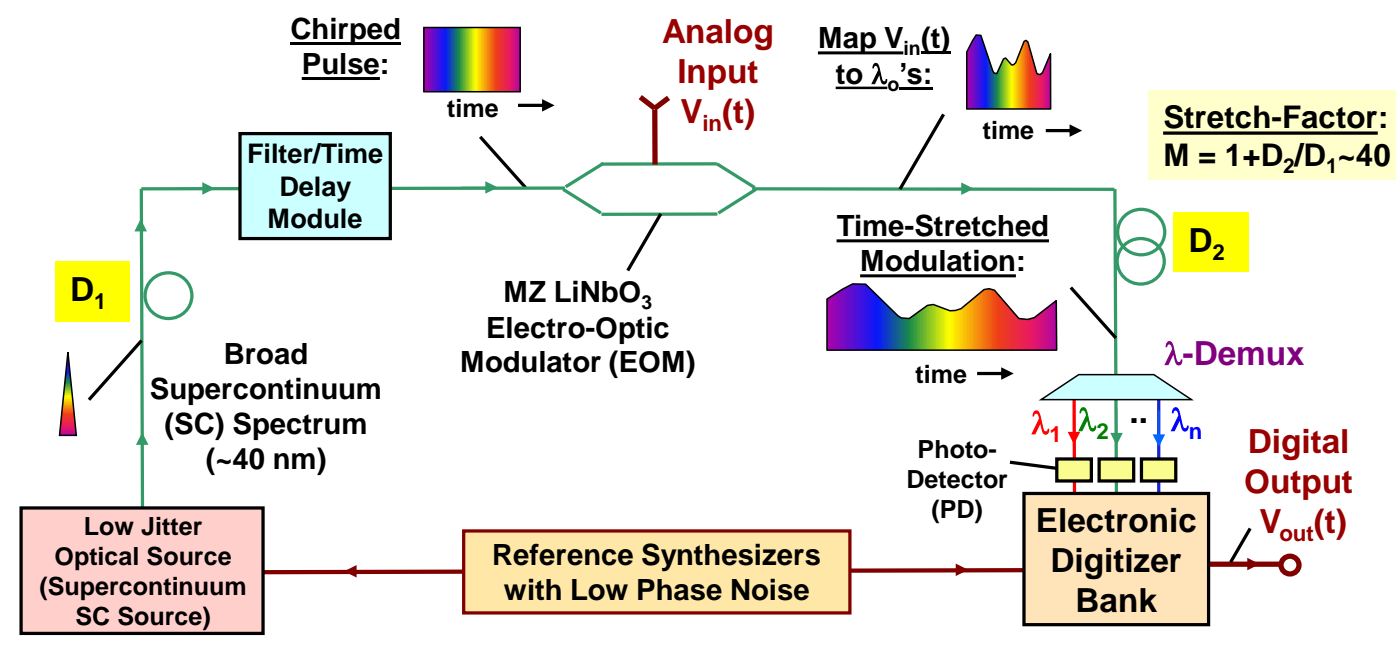

Fig.9 Schematic of a time-stretched (TS) photonic ADC system. The Filter/Time-Delay Module after $D_{1}$ provides an edge-overlap of the SC pulses (formed by spectral slicing with filters in the module) for Chstitching during post processing.

We then use a second dispersion element that has a large dispersion coefficient $\mathrm{D}_{2}$ to stretch the pulses - hence the modulation envelope - in the time-domain. For example, using a DCF with $\mathrm{D}_{1}=-50.5 \mathrm{psec} / \mathrm{nm}$ and a CFG with $\mathrm{D}_{2}=-2033 \mathrm{psec} / \mathrm{nm}$ as in Ref.48 (see Fig. 10), an analoginput at $\mathrm{f}_{\mathrm{in}}=10 \mathrm{GHz}$ was stretched to $\mathrm{f}_{\mathrm{TS}}=242 \mathrm{MHz}$ before digitization. The stretch-ratio $\mathrm{M}$, given by $M=1+D_{2} / D_{1}$, was $M=41.3$ in this example. After time-stretching, successive pulses in the ML pulse train would become overlapped in time. However, the time-stretched (TS) modulation envelope has been mapped to optical wavelength $\left(\lambda_{\mathrm{o}}\right)$. This allows us to demultiplex the analog-modulation into optical wavelength channels with use of optical filters or AWG demuxes. After the photodetected output of each optical wavelength channel (labeled $\lambda_{1} \ldots \lambda_{n}$ in Fig.9) is digitized, they are "stitched" back together during post-processing to form the digitized output $\mathrm{V}_{\text {out }}(\mathrm{t})$. As the ADC surveys of Ref. 38-39 show, a large ENOB can be attained for low input-signal frequencies because they are less sensitive to aperture-jitter. In addition, electronic ADC-chips that deliver a high ENOB for analog-inputs at low frequencies (e.g., ENOB 9.5 for 
$\left.\mathrm{f}_{\mathrm{s}} \sim 1-1.5 \mathrm{GHz}\right)$ are already available commercially. Therefore, the photonic time-stretch approach will enable a high frequency signal to be digitized with good SNDR via use of paralleled ADC-chips that sample at much lower rates.

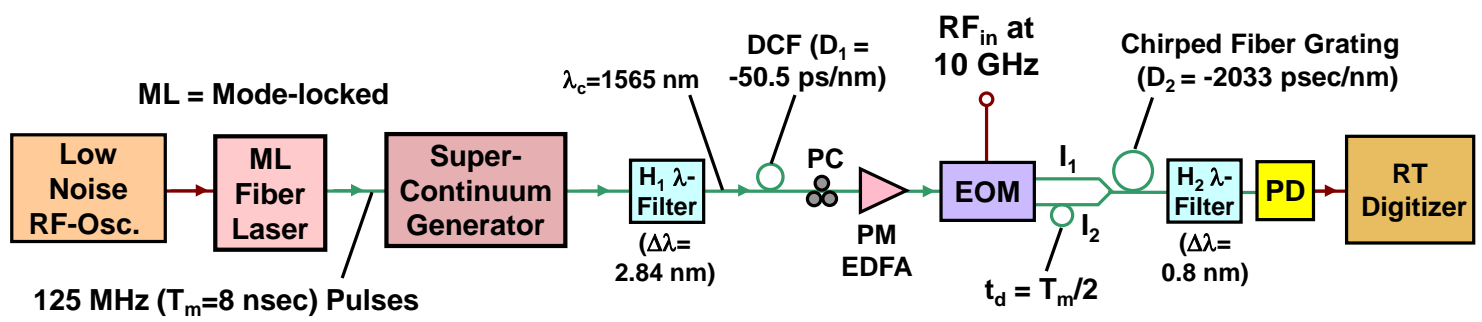

Fig.10 Schematic of the M=41 TS photonic ADC system [48] formed using an actively ML SC laser source with pulse repetition period of $\mathrm{T}_{\mathrm{m}}=8 \mathrm{nsec}$. In the figure, $\mathrm{PC}$ and $\Delta \lambda$ denote, respectively, polarization controller and $\mathrm{BW}$ of the filters $\left(\mathrm{H}_{1}, \mathrm{H}_{2}\right)$. A real time $(\mathrm{RT})$ digitzer that had an analog bandwidth of $16 \mathrm{GHz}$ was used to take the data of Fig.11.

To implement the time-stretch concept successfully, we have to take care of some details on component calibration as well as the post-processing of digitized data. First, we digitally linearize the optical modulation derived from the MZ EOM that has a raised cosine transfer curve, so that a high optical modulation index $m$ can be used to enhance the SNR of our photodetector (PD) output. This was accomplished with use of a MZ EOM that had complementary output arms $I_{1}$ and $I_{2}$ (see Fig. 10), and the arc-sine algorithm [42, 47]:

$$
\phi=\sin ^{-1}\left(\frac{I_{1}-I_{2}}{I_{1}+I_{2}}\right)
$$

In Eq.3, $\phi$ is the phase-modulation of the Mach Zehnder (MZ) EOM that is perfectly linear. Using a dual-output EOM and the arc-sine algorithm allowed us to use large modulation indices of $\mathrm{m} \sim 0.5-0.6$ (see Fig.11) and obtain a high signal-to-noise ratio (SNR). Furthermore, the combination also helped reject common mode noise such as relative intensity noise (RIN) from the SC source and Er-doped fiber amplifier (EDFA), as well as CFG ripples when a common time-stretch element $\mathrm{D}_{2}$ was used for $\mathrm{I}_{1}$ and $\mathrm{I}_{2}$ (see Fig.10). Second, we need to implement "time-shift corrections" [48] for the digitized data during post processing. Specifically, the group-delays of all the filters $\left(\mathrm{H}_{1}\right.$ and $\left.\mathrm{H}_{2}\right)$, as well as the dispersion elements $\left(\mathrm{D}_{1}\right.$ and $\left.\mathrm{D}_{2}\right)$ need to be characterized with either an optical vector network analyser (OVA) or a microwave network 
analyser (used in conjunction with a tunable laser). As described in Ref. 48-49, the transmission and group-delay data obtained from these spectral measurements allowed us to determine the stretch-ratio $\mathrm{M}$, map the filter-passbands from $\lambda$ to time, and estimate the time-shift corrections (incurred by nonlinear-stretching) for post-processing of the digitized data.

After applying time-shift corrections, we extracted the RF-modulation envelope from the digitized data of the photodetected pulses with Eq. 3. We then performed a least-square sine-fit to the sampled data lying within a time-window (see Fig.11a) that excluded pulse-edge effects such as ringing in the photodiode responses. From a sine-fit to the sampled data points, we obtained normalized amplitudes of the analog-input, as well as differences (fit-error) between the data and least-square fit for each pulse. The ENOB was then estimated with use of Eq.2.
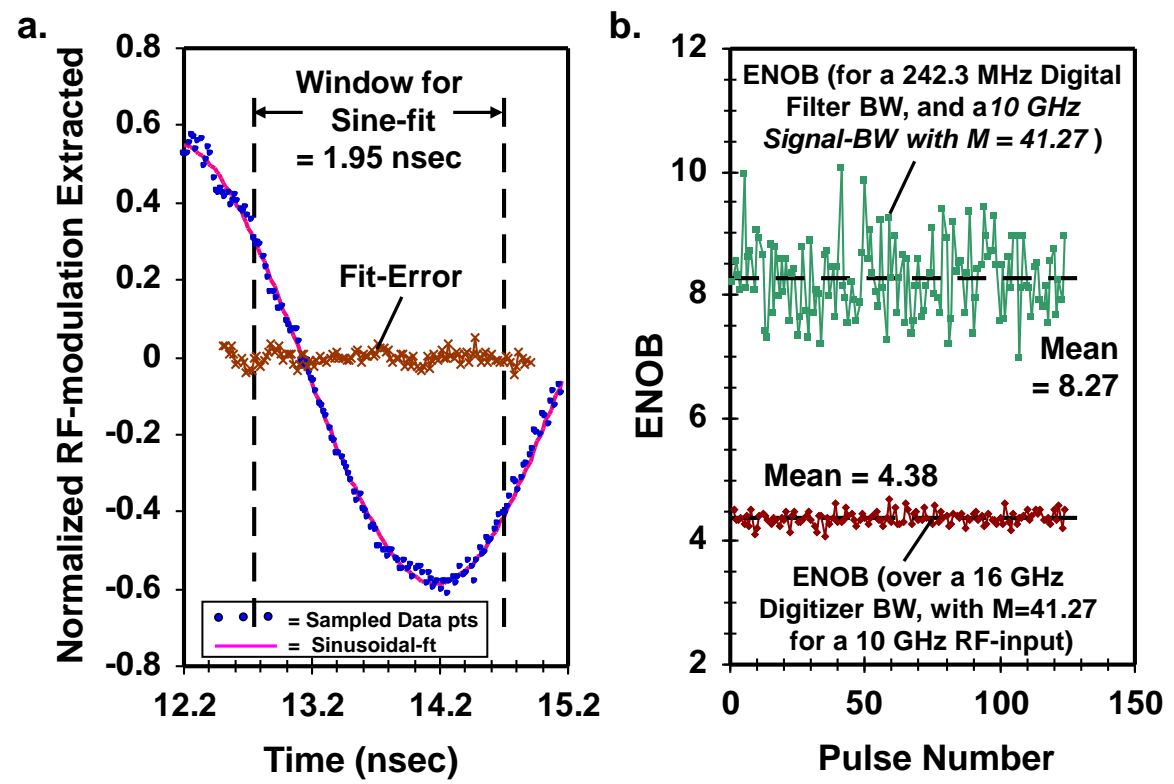

Fig.11 a. RF-Modulation extracted from the arc-sine algorithm for a $10 \mathrm{GHz}$ analog-input stretched to fTs = 242.3 MHz, and its sine-fit used for ENOB evaluation [48] b. ENOB (lower trace, with a mean value of 4.38) obtained for a $16 \mathrm{GHz}$ noise-bandwidth of the RT digitizer, and the ENOB (upper trace, with a mean value of 8.27) obtained after digital filtering with a lowpass-filter designed to have a cutoff at $f_{T S .}$

The bottom trace of Fig. $11 \mathrm{~b}$ shows that an ENOB of 4.38 was obtained for the $\mathrm{f}_{\mathrm{TS}}=242.3 \mathrm{MHz}$ TS signal, over the $16 \mathrm{GHz}$ noise BW of the real time (RT) digitizer. Over the above noise BW, the RT digitizer could deliver an ENOB of 5.35-5.8 for $250 \mathrm{MHz} C W$-inputs with RF power levels of -17 to $-2.5 \mathrm{dBm}$. We then applied a digital (Matlab) low-pass filter with a passband 
width of $\mathrm{f}_{\mathrm{TS}}$ to the fit-error. With the reduction in noise bandwidth from $16 \mathrm{GHz}$ to $\mathrm{f}_{\mathrm{TS}}$, we should enhance the SNDR by at least $18 \mathrm{~dB}$ for a white noise spectrum, with a corresponding $\triangle$ ENOB of 3. For the data shown in Fig.11b, the ENOB enhancement ( $\triangle$ ENOB) after the above low-pass filtering was actually 3.89, which indicated that some high frequency spurs (e.g., CFG ripples or higher order harmonic distortions) were effectively removed by the low-pass filtering [50]. Specifically, we obtained, as an average, an ENOB of 8.27 (over a noise $\mathrm{BW}=\mathrm{f}_{\mathrm{TS}}$ ) for the 124 pulses in the data set. In our work, the achieved ENOB of 8 or better for a $10 \mathrm{GHz}$ analoginput had been obtained consistently for DC-photocurrents $\mathrm{I}_{\mathrm{dc}}$ of $\sim 1.5 \mathrm{~mA}$ and $\mathrm{m} \sim 0.5-0.6$. By stitching together the RF-modulation (analog-input) from wavelength channels - a subset of the total number of channels required for continuous time A/D conversion - that were de-serialized for photodetection and $\mathrm{A} / \mathrm{D}$ conversion, we also achieved an ENOB $\geq 8$ for a $10 \mathrm{GHz}$ signalinput, using as backend quantizers: (i) a RT digitizer, as well as (ii) COTS ADC chips that sampled at the rate of $1 \mathrm{Gs} / \mathrm{s}[51]$.

Finally, we also have to take into account of dispersion induced penalty (DIP) in designing a TS ADC system. As in conventional RF-photonic links that use double-sideband (DSB) modulation, the upper and lower sidebands can become $\pi$ out-of-phase over long propagation distances due to the $2^{\text {nd }}$ order dispersion coefficient, i.e. $\partial v_{g}(\omega) / \partial \omega$, where $\operatorname{vg}(\omega)$ is the group velocity of the dispersion elements at optical frequency $\omega$. The DIP will cause a null in the received signal when the photodetector currents due to the upper and lower sidebands interfere destructively. In a TS ADC system that has a time-stretch ratio M, the RF transfer function that models the DIP is given by:

$$
H_{R F}\left(f_{\text {in }}\right)=\cos ^{2}\left(\phi_{D I P}\right)=\cos ^{2}\left(\pi \frac{\lambda^{2}}{c} D_{1} f_{\text {in }}^{2}\right)
$$

where $f_{\text {in }}$ is the frequency of the analog-input [46]. Fig. 12 shows a plot of the $\operatorname{HRF}_{R F}\left(f_{\text {in }}\right)$, for $\left|\mathrm{D}_{1}\right|=200 \mathrm{psec} / \mathrm{nm}$ and $\lambda \sim 1550 \mathrm{~nm}$. Notice from Eq. 4 that although the $2^{\text {nd }}$ dispersion element (with coefficient $\mathrm{D}_{2}$ ) is responsible for delivering a large TS ratio (i.e., $\mathrm{D}_{2}>\mathrm{D}_{1}$ typically), $\phi_{\text {DIP }}$ in a TS photonic ADC system is set by the first dispersion element (with coefficient $\mathrm{D}_{1}$ ). This is another artifact of the bandwidth-compression obtained via use of time-stretching. From Eq. 4, we obtain the RF-frequency fin where the first null is located as: $f_{n u l l}=\left[c /\left(2 \lambda^{2} D_{1}\right)\right]^{0.5}$. In 
particular, the expression for $f_{\text {null }}$ shows that $D_{1}$ must be small enough so that $f_{\text {null }}>f_{\text {in }}$. On the other hand, given a spectral width $\Delta \lambda_{\mathrm{sc}}$ for the SC, $\mathrm{D}_{1}$ must be sufficiently large so that the product $\mathrm{D}_{1} \Delta \lambda_{\mathrm{sc}}$ is slightly larger than the interpulse period $\mathrm{T}_{\mathrm{m}}$ to accomplish continuous-time operation. Therefore, in addition to the TS ratio M, there are design tradeoffs for the parameters $\mathrm{D}_{1}, \Delta \lambda_{\mathrm{sc}}$, and $\mathrm{T}_{\mathrm{m}}$ in a continuous-time TS ADC system.

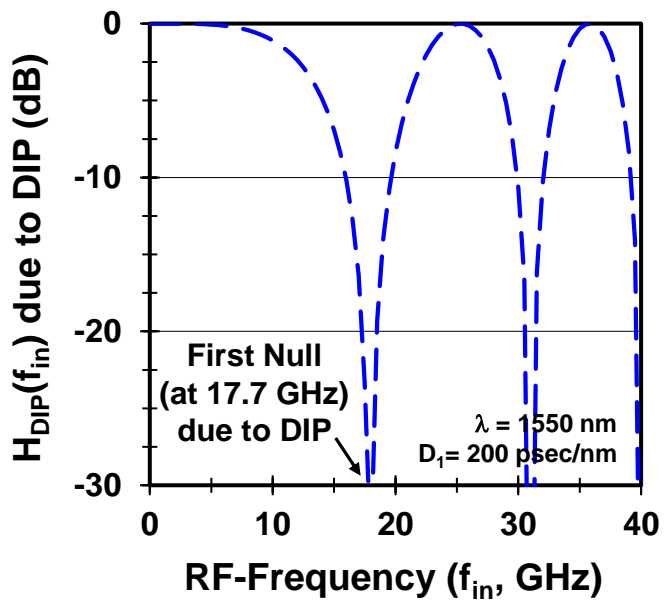

Fig. 12 Plot of the transfer function $\mathrm{HDIP}_{(\mathrm{in})}$ [Eq. 4] modeling the dispersion induced penalty (DIP) in a TS photonic ADC system

In summary, the use of wideband optical dispersion and highly stable ML pulse trains have helped electronic quantizers overcome the "Walden Wall" imposed by "aperture uncertainty" during sampling. The achievement of $8 \mathrm{ENOB}$ for a $10 \mathrm{GHz}$ signal-input attests to the stability of a photonic ADC system. The photonic ADC subsystems described are compatible with antenna remoting architectures that allow the low loss distribution of clock signals via fibers to multiple ADCs. Finally, our photonic $\Delta \Sigma$ ADC fabricated with integrated photodetectors and feedback-loops operating in the multi-GHz range demonstrated the performance enhancements obtained via optoelectronic integration.

\section{RF-Photonic Filtering}

The interests in developing reconfigurable RF-photonic filters that can accomplish the preprocessing of RF-signals over a wide IBW are well known [6, 16, 52-63]. We illustrate, in Fig.13, how these RF-photonic filters can serve as "bandwidth transformers" in an apertureremoted, multi-function antenna system that incorporates WDM technologies. As shown, a 
wideband incident $\mathrm{RF}$-signal $\left(\mathrm{RF}_{\text {in }}\right)$ can be modulated simultaneously onto multiple optical carriers with use of an EOM. Using an agile RF-photonic filter that can operate over a wide IBW (e.g., IBW $\geq 10 \mathrm{GHz}$ ), the modulated RF-signal is filtered to narrower frequency-segments (e.g., $\leq 500 \mathrm{MHz}$ ), so that they can be digitized with high resolution for digital signal processing (DSP). Furthermore, the filtered signal remains in the optical domain. Therefore, it can be distributed with low loss, EMI-immune fibers to multiple receivers at different physical locations.

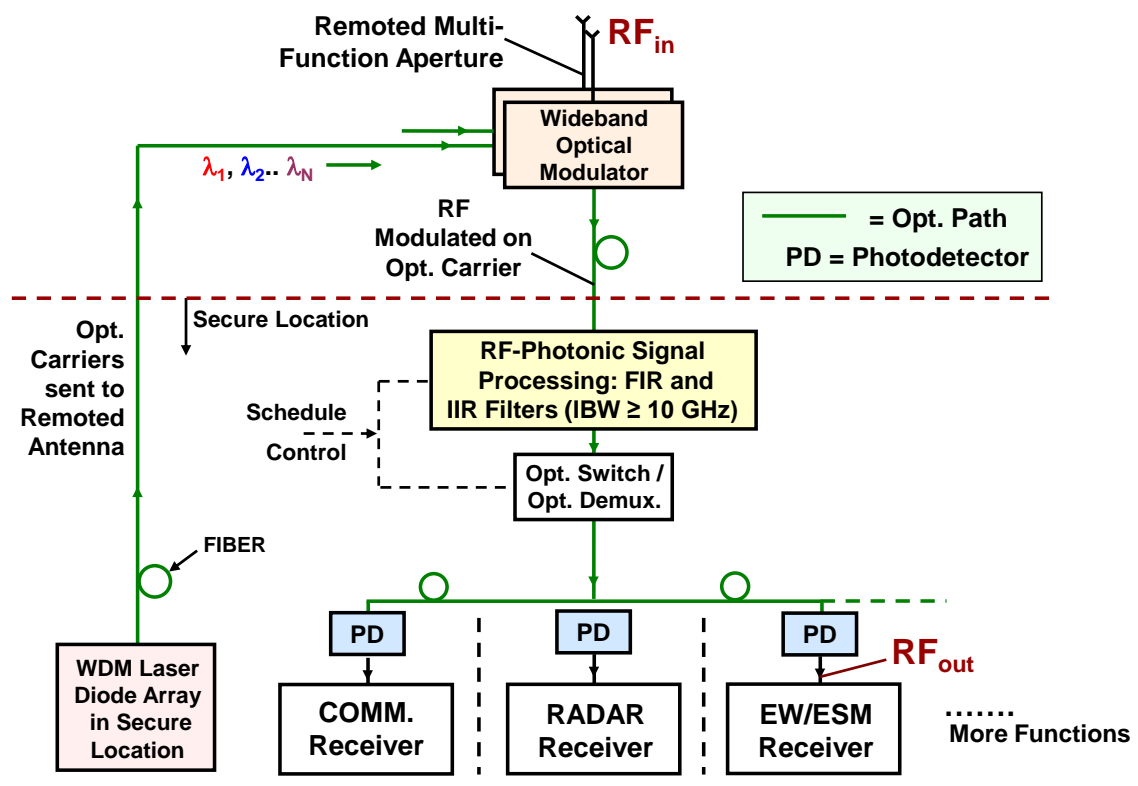

Fig.13 RF-photonic filters in an aperture-remoted microwave antenna system. After filtering, the filteroutput that remains in the optical domain is distributed to multiple physical locations for photodetection, high resolution A/D conversion, and DSP.

In Sections 4.1 and 4.2, we discuss, respectively, the formation of finite impulse response (FIR) and infinite impulse response (IIR) RF-Photonic filters.

\subsection{RF-Photonic FIR Filters}

Many approaches [16, 52-57] have been demonstrated for the formation of RF-photonic FIR filters. To discuss the salient features of these filters, we pick the configuration depicted in Fig. 14 [57]. As commented in [52], the multiple-wavelength WDM delay-line scheme is a "straightforward" approach to eliminate "coherence limitations" in photonic signal processors. We project that advancements in III-V compound semiconductor and EOM technologies will 
make this approach even more attractive (e.g., in form-factor) for system insertion. As shown, the outputs of 20 DFB lasers were multiplexed with an AWG-mux to form an optical input for the MZ EOM. The input-RF signal ( $\left.R F_{\text {in }}\right)$ to be filtered was impressed on all 20 optical carriers,

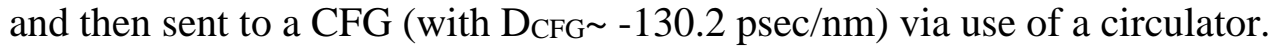

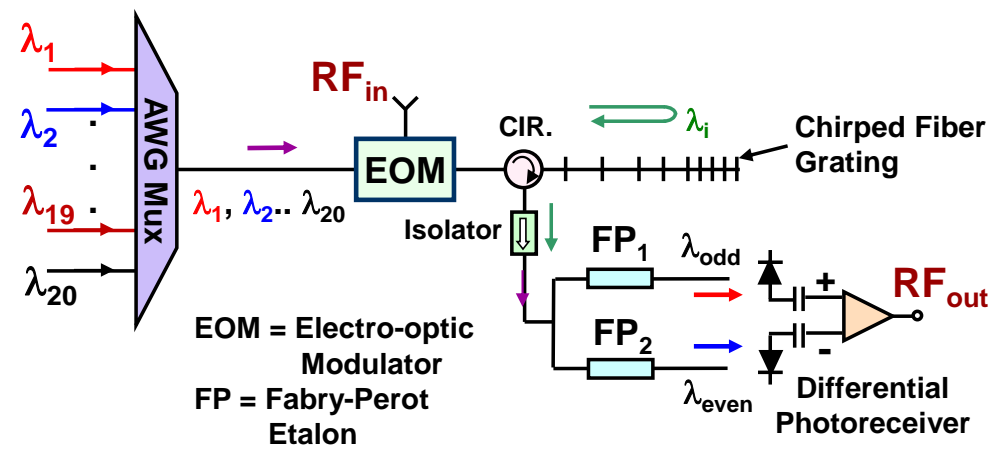

Fig.14 Schematic of bipolar FIR RF-photonic filter implemented using WDM technologies, a length of chirped fiber grating (CFG) and a differential photoreceiver [57]

We take note that today's III-V fabrication technology had already demonstrated [64] the monolithic integration of 40 AWG-multiplexed DFB lasers on a single InP-chip, along with semiconductor amplifiers (SOAs) for power-equalization in each $\mathrm{Ch}$. The lasing wavelengths of these DFB lasers are aligned along the ITU-grid, with Ch-separations of $\Delta \mathrm{f}_{\mathrm{opt}}=50 \mathrm{GHz}$.

Therefore, they can serve as monolithic WDM transmitters for the filter of Fig.14. As mentioned in Section II, AWGs demonstrate extremely low multiplexing losses even for a large number of $\lambda$-Chs because they are interference-based optical devices. For this reason, the mux/demux attributes of AWGs (for RFin) are not easy to emulate with multi-channel power combiners/splitters developed in the RF-regime. With further improvements in link noise-figure, WDM technologies can be adopted to form a signal-routing backbone that incorporates low loss multiplexers/demultiplexers for accomplishing a variety of signal-processing functions in microwave systems.

As described in Ref. 57, we generated a series ( $\mathrm{N}=20)$ of polarity-interlaced taps with help of a differential photoreceiver and two inline Fabry-Perot etalons whose passbands were offset from each other by $100 \mathrm{GHz}$. The ITU-grid $\left(\Delta \mathrm{f}_{\mathrm{opt}}=100 \mathrm{GHz}\right)$ aligned emission wavelengths of our DFB lasers generated tap-delays $\left(\tau_{\mathrm{d}}\right)$ of $103.5 \mathrm{psec}$, which corresponded to an equivalent 
sampling rate of $\mathrm{f}_{\mathrm{s}} \sim 10 \mathrm{GHz}$ in a digital processor. Hence, the FIR filter we formed has a large free spectral range (FSR) or an IBW of $\sim 10 \mathrm{GHz}$. The bipolar taps thus formed also enabled our first passband to become offset from DC, and be located at $f_{c}=1 /\left(2 \tau_{d}\right)$ in accordance with Eq. 5 [57]:

$$
\left|H\left(\omega_{m}\right)\right|^{2}=\left|\sum_{n=0}^{N-1} a_{n} e^{-j n \omega_{m} \tau_{d}}\right|^{2}
$$

In Eq.5, $\omega_{\mathrm{m}}$ is the RF-frequency and $\mathrm{a}_{\mathrm{n}}=-\mathrm{a}_{(\mathrm{N}-1)-\mathrm{n}}$ for a even number of taps $\mathrm{N}$. As reported in [57], the antisymmetry of our filter-taps also delivered a linear phase response. Fig.15a shows the optical spectrum of 20- $\lambda$ taps that had been apodized in amplitude with Dolph-Chebyshev coefficients. In our work [57], the amplitude tapering was accomplished by controlling the power-output of the DFB lasers. Fig.15b shows the RF-response measured for the above apodized taps. As shown, the Dolph-Chebyshev FIR filter demonstrated a side-lobe suppression ratio (SLSR) of $32.2 \mathrm{~dB}$, along with a $-1 \mathrm{~dB}$ passband width (PBW) of $\Delta \mathrm{f}=463 \mathrm{MHz}$. The passband's center-frequency $\left(\mathrm{f}_{\mathrm{c}}\right)$ of $4.8 \mathrm{GHz}$ was in excellent agreement with that estimated from our model for a $\tau_{\mathrm{d}}$ of $103.5 \mathrm{psec}$.
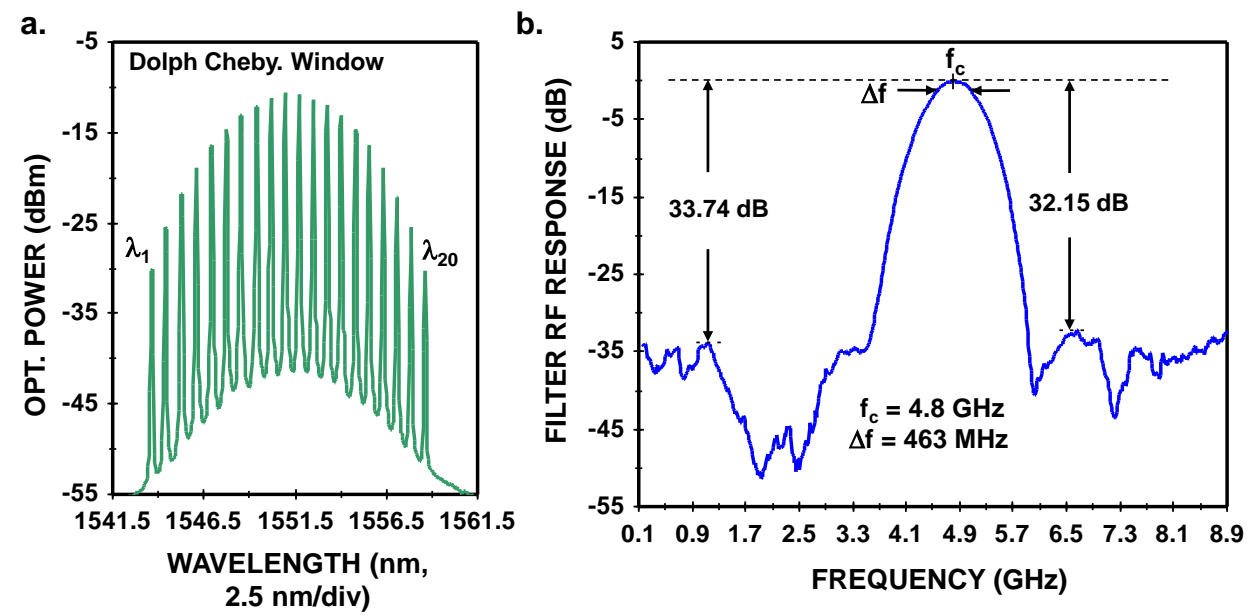

Fig.15 a. Optical spectrum of a WDM comb windowed to form a 20-tap Dolph-Chebyshev filter. b. RF response of the FIR filter formed with use of the tap-apodization shown in Fig.15a. In the figure, $f_{c}$ and $\Delta f$ denote, respectively, the center frequency of passband and its -1 dB PBW. 
In Ref. 57, we used a $\mathrm{LiNbO}_{3} \mathrm{MZ}$ EOM for impressing $\mathrm{RF}_{\text {in }}$ onto the optical carriers $\lambda_{\mathrm{i}}$ $(i=1 . .20)$. We take note that the half-wave voltage $\left(\mathrm{V}_{\pi}\right)$ of these $\mathrm{LiNbO}_{3} \mathrm{MZ}$ EOMs had been reduced to $\mathrm{V}_{\pi}<1.5 \mathrm{~V}$ at multi-GHz frequencies [65], which would help lower the link noise figure. Alternatively, substrate-removed InP-EOM with $\mathrm{V}_{\pi}<1 \mathrm{~V}$ [66] can be used, but they have lower optical power handling capabilities. The transmitter and receiver shown in Fig.14 can be integrated on an InP substrate or heterogeneously on Si. With further development of integration technologies for low $\mathrm{V}_{\pi}$ modulators and the incorporation of link linearization (e.g., via predistortion or digital post-processing approaches [67]), the above transversal filter has the potential to be implemented compactly, so that it can meet the noise figure, SFDR and formfactor expectations of microwave system integrators.

Finally, we discuss the reconfiguration of our FIR filter. By routing (optically switching) the incident $\lambda_{\mathrm{i}}$ 's from the above $\mathrm{CFG}\left(\mathrm{D}_{\mathrm{CFG}} \sim-130.2 \mathrm{psec} / \mathrm{nm}\right.$ ) to a second one with $\mathrm{D}_{\mathrm{CFG}} \sim-97.6$ $\mathrm{psec} / \mathrm{nm}$, we were able to re-center the filter passband, as expected, from $4.8 \mathrm{GHz}$ to $6.33 \mathrm{GHz}$. We show, in Fig.16, the additional potential to reconfigure filter passbands via the dropping of wavelengths $\lambda_{\mathrm{i}}$ or the switching of antisymmetric bipolar-taps to unipolar-taps.

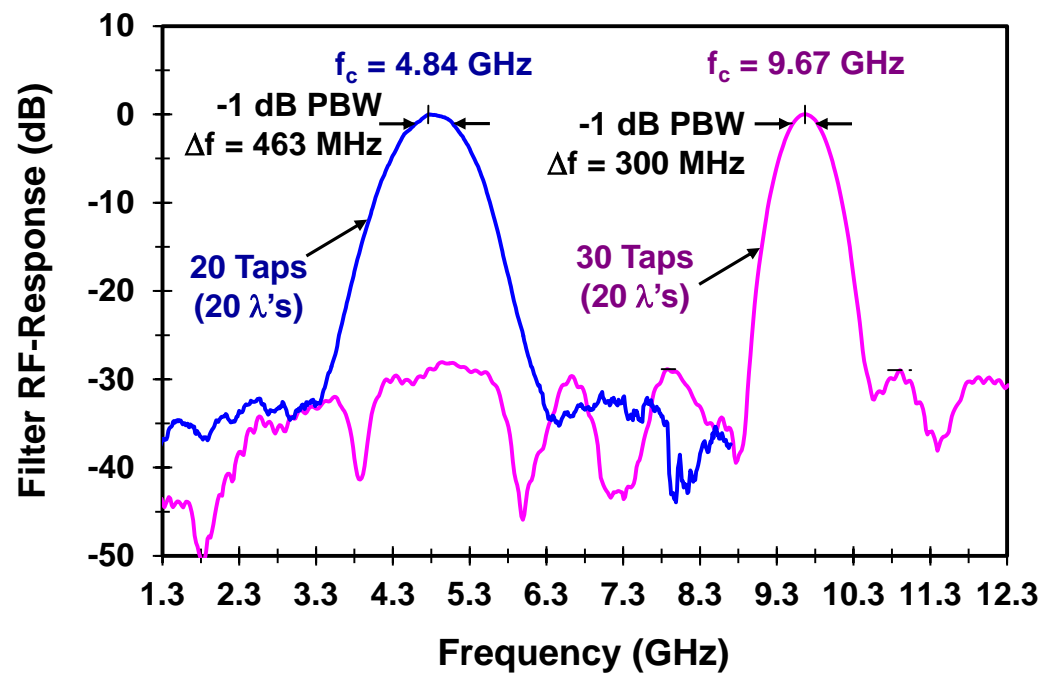

Fig.16 Reconfiguration of the filter-passband from a 30-tap unipolarity ( $\lambda$-reused) FIR filter to a 20-tap bipolar filter. Both filters were formed by adopting Dolph-Chebyshev coefficients for tap-apodization.

In the figure, the unipolar-filter (centered at $f_{c}=9.67 \mathrm{GHz}$ ) with the narrower bandpass was obtained by using a $\lambda$-reuse scheme [68] that generated 30 taps from 20 incident wavelengths. 
When we switch back to the bipolar 20-tap configuration of Fig.14, the filter is re-centered to $\mathrm{f}_{\mathrm{c}}=4.84 \mathrm{GHz}$, but with a wider passband.

In summary, the interests in developing RF-photonic FIR filters stem from: its potential to serve as a reconfigurable "bandwidth transformer" between a wideband RF-frontend and high resolution backend-ADCs, its high equivalent sampling rates $\left(=1 / \tau_{\mathrm{d}}\right)$, and the low loss optical distribution of filter-outputs. Further improvements in enhancing their link gain and SFDR, along with the realization of chip-scale form-factors, will lead to system insertion opportunities that demonstrate the above merits.

\subsection{RF-Photonic IIR Filters}

While almost all of the FIR filters discussed (and referenced) above operate in the optical incoherent regime, the rapid development of chip-integrable microresonators (MR, e.g., microrings, racetrack resonators) led to the demonstration of many coherent IIR filters fabricated on InP or Si substrates [11, 58-61]. The modeling and design of these IIR filters were reported much earlier [62-63]. In this Section, we provide an introduction to the DSP approach of synthesizing IIR optical filters, and describe examples of filters formed via the cascading of unit cells.

A DSP approach can, in general, be adopted to model the transfer functions of optical resonators, including Fabry-Perot (FP) resonators. Fig.17a and b show, respectively, a FP resonator and a MR coupled to its incident- and drop-WG. A one-to-one correspondence can be established between: (i) the reflection $\mathrm{E}_{\mathrm{r}}$ of the FP resonator and the throughput $\mathrm{E}_{\mathrm{e}}$ of the MR, as well as (ii) the transmission $\mathrm{E}_{\mathrm{t}}$ of the FP resonator and the MR drop-output $\mathrm{E}_{\mathrm{d}}$. In addition, the reflections $r_{i}(i=1,2)$ inside the FP resonator correspond to the internal ("bar-port") coupling coefficient $\mathrm{t}_{\mathrm{mi}} *(\mathrm{i}=1,2)$ within the MR. 
a.

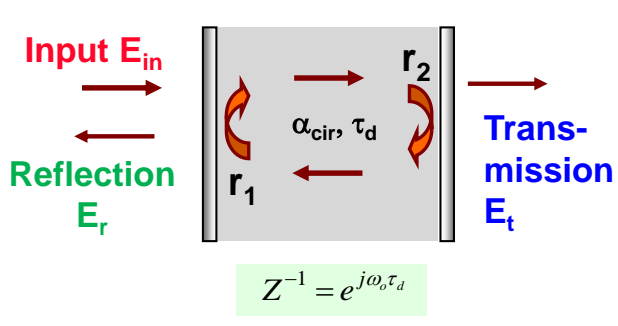

b.

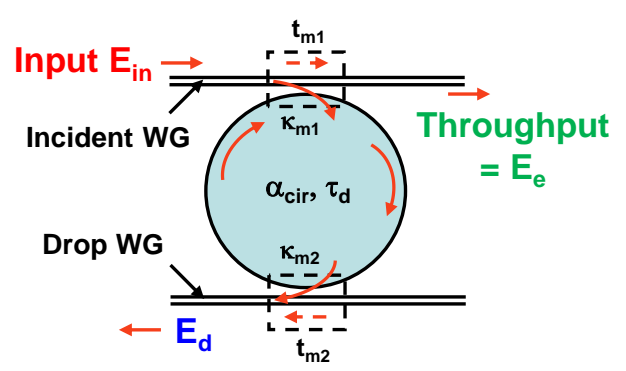

c.

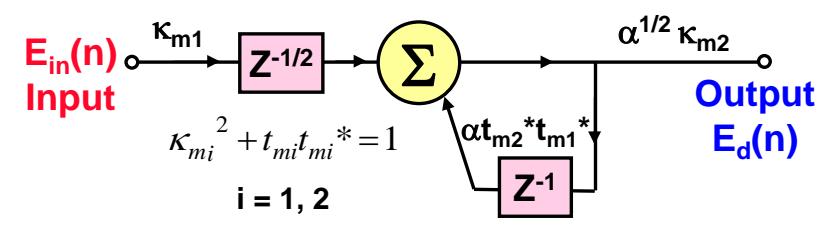

Fig.17 a. Fabry-Perot (FP) etalon b. Microresonator (MR) evanescently coupled to incident and drop waveguides c. Digital filter model of the drop-output for the MR of Fig.17b

Using $z^{-1}=e^{j \omega_{o} \tau_{d}}$, the outputs $\mathrm{E}_{\mathrm{d}}$ and $\mathrm{E}_{\mathrm{e}}$ of the MR can be written as [69-70]:

$$
\begin{aligned}
& \frac{E_{d}}{E_{i}}=\frac{\sqrt{\alpha_{c i r}} \kappa_{m 1} \kappa_{m 2} z^{-1 / 2}}{1-\left(\alpha_{c i r} t_{m 2} *\right) t_{m 1} * z^{-1}} \\
& \frac{E_{e}}{E_{i}}=\frac{t_{m 1}-\left(\alpha_{c i r} t_{m 2} *\right) z^{-1}}{1-\left(\alpha_{c i r} t_{m 2} *\right) t_{m 1} * z^{-1}}
\end{aligned}
$$

where $\alpha_{c i r}$ is the round-trip transmission coefficient inside the MR. In addition, the following relationship holds for $\kappa_{m i}$ and $t_{m i}$ at coupling junctions: $\kappa_{m i}{ }^{2}+\left|t_{m i}\right|^{2}=1$, where $\mathrm{i}(=1$ or 2$)$ denote the incident and drop coupling-junctions respectively. From Eq. 7, we see that the throughput of a MR can be modeled as a first order IIR filter that has one pole and one zero. Using Eq.6 and Eq.7, higher order optical filters can be synthesized by cascading unit cells and adopting DSP pole/zero approaches. For example, Fig.17c shows a Z-domain representation of the drop-output $\mathrm{E}_{\mathrm{d}}$. Since $\tau_{\mathrm{d}}$ is typically $<20 \mathrm{psec}$, the IIR filters thus formed are optical devices that exhibit a much higher equivalent sampling rate $\mathrm{f}_{\mathrm{s}}\left(=1 / \tau_{\mathrm{d}}\right)$ than present electronic digital filters.

The optical coupling parameters (i.e., $\kappa_{m}$ ) of these MRs and their relationship to the qualityfactor (Q) and extinction ratio (ER) of the measured resonances had been well characterized [70- 
71]. Fig.18 shows the throughput and drop resonance spectrum measured at critical coupling for a silica microdisk formed via projection lithography on a Si-substrate. As shown, the loaded-Q, Qtotal of the MR, was measured to be $\sim 1.56 \times 10^{6}$. The data of Fig. 18 also shows that the measured drop efficiency at critical (cr) coupling was $\sim 83 \%$, which was in good agreement with a $D_{\max }$ of $88 \%$ estimated from the equation [71]:

$$
\left.D_{\max }\right|_{c r}=1-\frac{2 Q_{\text {total }}}{Q_{0}}
$$

As Eq.8 shows, the excellent drop efficiency we obtained was due to the high intrinsic-Q, Qo[72] of the silica MR. These measurements also indicate that when MRs (e.g., Si MR) are used to form IIR filters, their $Q_{o}$ needs to be maximized, otherwise cascades of MRs with aligned resonances would have to be implemented to achieve a high passband rejection-ratio.

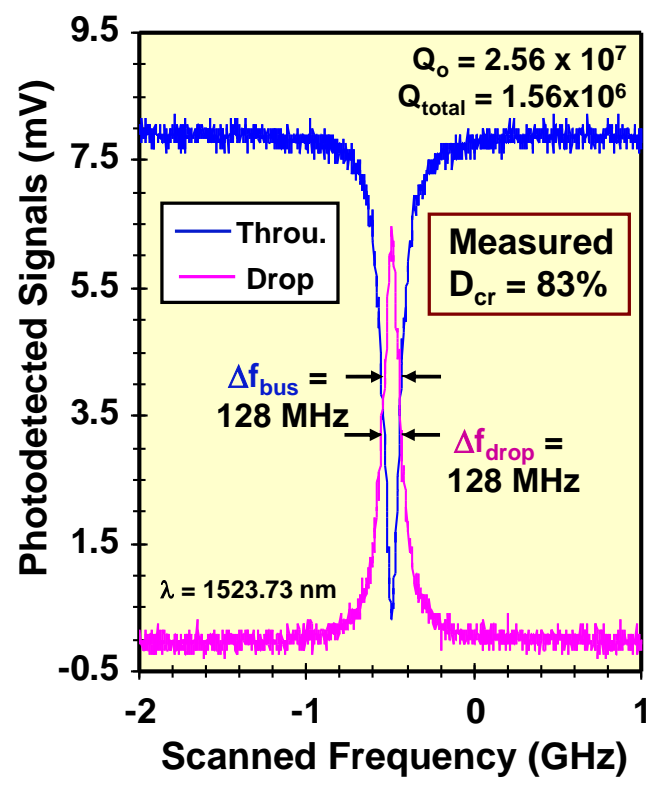

Fig.18 Measured throughput (Throu.) and Drop-output (Drop) of a silica/Si MR with an intrinsic-Q $\left(Q_{0}\right)$ of $Q_{0}=2.56 \times 10^{7}[72]$. The evanescent coupling to the MR was accomplished with use of fiber-tapers.

Fig.19 shows a second order unit cell formed by a MZ interferometer (MZI) where two microresonators $\left(\mathrm{MR}_{\mathrm{i}}, \mathrm{i}=1,2\right)$ are coupled to an input directional coupler $(\mathrm{R}(\theta))$ and an output directional coupler $(\mathrm{R}(\phi))$. 


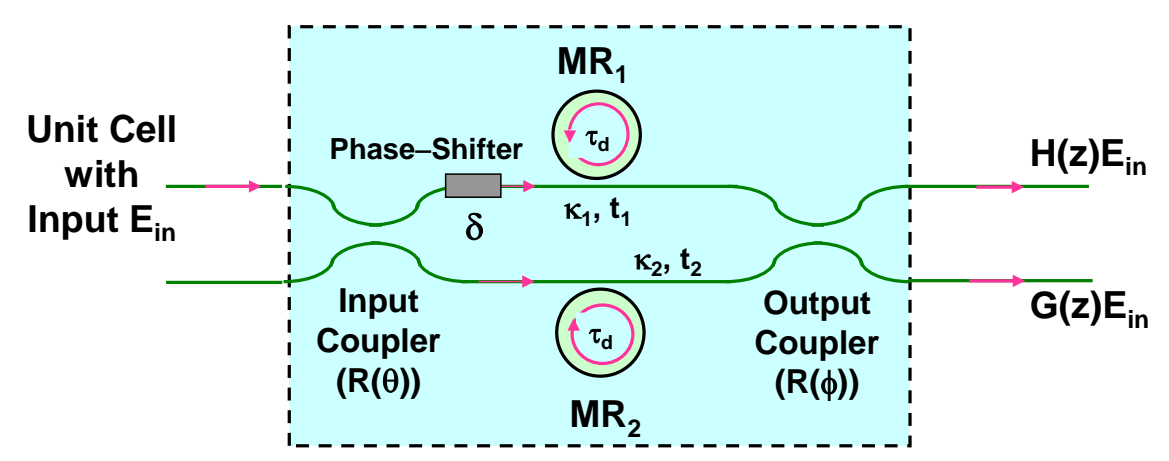

Fig.19 Second-order unit cell formed by coupling microresonators $M R_{1}$ and $M R_{2}$ to an input and an output directional coupler. A phase-shifter $\delta$ provides tuning of the relative phase between the two arms.

In this unit cell, only the throughput port of the MRs are coupled to the output direction coupler $\mathrm{R}(\phi)$. $\mathrm{H}(\mathrm{z})$ and $\mathrm{G}(\mathrm{z})$ of Eq.9 below are then obtained as transfer functions for the two outputs of the directional coupler $\mathrm{R}(\phi)$ :

$$
\left(\begin{array}{l}
H \\
G
\end{array}\right)=R(\phi)\left(\begin{array}{cc}
1 & 0 \\
0 & e^{i \delta}
\end{array}\right)\left(\begin{array}{cc}
H_{1} & 0 \\
0 & H_{2}
\end{array}\right) R(\theta)
$$

In Eq.9, $\delta$ is the phase-shift between the two MZI arms, $\alpha_{i}$ the round trip transmission coefficient within microresonator $\mathrm{MR}_{\mathrm{i}}$, and

$$
\begin{aligned}
& R(\psi)=\left(\begin{array}{ll}
\cos \psi & j \sin \psi \\
j \sin \psi & \cos \psi
\end{array}\right) \quad \psi=\theta \text { or } \phi \\
& H_{i}(z)=\frac{t_{i}-\alpha_{i} z^{-1}}{1-\alpha_{i} t_{i}^{*} z^{-1}}
\end{aligned}
$$

In particular, $\theta$ and $\phi$ of Eq.10 are parameters that model the optical coupling (split-ratio) from the two input-ports of the directional couplers to their two output-ports. Both directional couplers $[R(\theta)$ and $R(\phi)]$ are assumed to have no excess loss in this first-order model. From Eq. 9-11, we see that the outputs $\mathrm{H}(\mathrm{z})$ and $\mathrm{G}(\mathrm{z})$ are second order transfer functions that have two poles and two zeros. When $\alpha_{\mathrm{i}}=1$, Eq.11 models an all-pass filter whose poles and zeros form reciprocal conjugate pairs [63]. Eq.11 also shows how the internal loss (quantified by $\alpha_{i}$ ) restricts the magnitude of the poles $\mathrm{p}_{\mathrm{i}}\left(\left|\mathrm{p}_{\mathrm{i}}\right|=\left|\alpha_{\mathrm{i}} \mathrm{t}^{*}\right|\right)$ inside the $\mathrm{Z}$-domain unit circle. In filter synthesis, this pole-magnitude restriction could limit the band-rejection ratio of filters formed by 
cascading the above unit cell. This is a fundamental difference between the synthesis of optical IIR filters and digital filters.

One approach to design a filter that can meet the specifications (e.g., PBW, band rejection ratio) of a targeted filter response is to: (i) design a digital filter with the coefficients of "namedfilters" (e.g., Butterworth, Chebyshev, or Elliptic filters), and then (ii) solve for the optical parameters:, $\mathrm{t}_{\mathrm{i}}, \alpha_{\mathrm{i}}, \theta, \psi$ and $\delta$ from equations (given in [73]) that relate them to the coefficients of $H(z)$. Taking the transfer function $H(z)$ for the upper-output of $R(\phi)$, we have:

$$
H(z)=\frac{1+B_{1} z^{-1}+B_{2} z^{-2}}{1+A_{1} z^{-1}+A_{2} z^{-2}}
$$

where $B_{i}, A_{i}(i=1,2)$ are functions of the variables $t_{i}, \alpha_{i}, \theta, \psi$ and $\delta$. Fig.20 shows the passbands of a filter H(z) synthesized [73] by cascading two of the unit cell shown in Fig.19, and using Elliptic filter coefficients. As shown, the filter response $\left(\tau_{\mathrm{d}}^{-1}=50 \mathrm{GHz},\left|\alpha_{\mathrm{i}}\right|=0.98\right)$ can be tuned from $3 \mathrm{GHz}$ to $8 \mathrm{GHz}$ by controlling the phase of $\alpha_{\mathrm{i}}$. The $-1 \mathrm{~dB}$ PBW for the two-cell cascade that uses a 1:1 split-ratio (i.e., $\theta=\phi=\pi / 4$ ) for its input and output couplers is $\sim 400 \mathrm{MHz}$.

a.

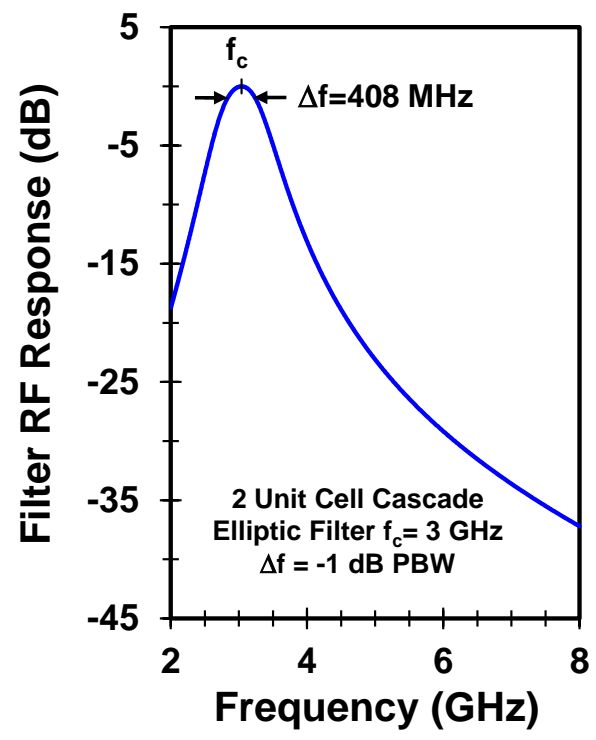

b.

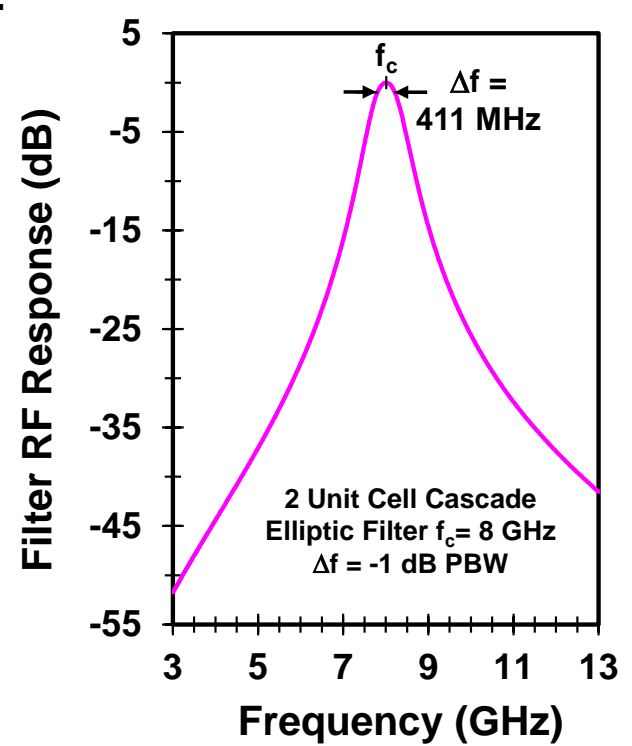

Fig.20 Bandpass filter formed by cascading two of the unit cell shown in Fig.19: a. Center $f_{c}$ of passband tuned to $3 \mathrm{GHz}$ b. Center $\mathrm{f}_{\mathrm{c}}$ of passband tuned to $8 \mathrm{GHz}[73]$ 
The above round-trip transmission $\alpha_{i}$ with $\left|\alpha_{i}\right|=0.98$ corresponds to an intrinsic $Q_{o}$ of $\sim 6.4 \times 10^{5}$ for $\tau_{\mathrm{d}^{-1}}=50 \mathrm{GHz}$ and $\lambda \sim 1550 \mathrm{~nm}$. Although the $\mathrm{Q}_{\mathrm{o}}$ of Si MRs that provides tunability has improved substantially in recent years, $\mathrm{Si}$ MRs that demonstrate the above $\mathrm{Q}_{\mathrm{o}}$ is still a challenge to fabricate when integrated with other components in a PIC. A new approach [74] that optimizes the filter coefficients according to physical constraints imposed by the optical components - including the MR internal loss that sets its $Q_{0}$ - could then be adopted as an alternate design approach. Although the second order unit cell shown in Fig.19 has not yet been fabricated, similar integrated filter structures have been demonstrated using a variety of WG technologies [75]. Many of these devices had demonstrated optical insertion losses that were dominated by high fiber-chip coupling losses. Using dielectric waveguides with more optimal fiber-coupling, a double-ring Mach-Zehner interleaver had demonstrated [76] a fiber-to-fiber insertion loss as low as $-2.2 \mathrm{~dB}$ at its passbands, with an estimated fiber-coupling loss of -1.2 to $1.5 \mathrm{~dB}$.

In summary, coherent photonic IIR filters can be realized in the form PICs. Compared with digital filters, they possess a much higher equivalent sampling rate $\mathrm{f}_{\mathrm{s}}$. A narrow PBW and a high band-rejection ratio can be obtained by enhancing the MR $Q_{0}$, and the optimization of filter coefficients.

\section{RF-Photonic Links}

In this Section, we provide a short discussion on RF-photonic links. Many excellent reviews had been written on this topic $[3,12,36-37]$, we focus on the more recent developments. Specifically, the recent progress in fabricating high-linearity and high-power photodetectors (PDs) of various uni-traveling carrier UTC designs [77-78] have led to the demonstration of many RF-photonic links that possessed positive gain $(\mathrm{G})$ and low noise figures $(\mathrm{NF})$ that were $\leq 10 \mathrm{~dB}$ [79-80]. Usng PDs that could maintain linearity at DC-photocurrents ( $\mathrm{Idc}_{\mathrm{dc}}$ as high as 4080mA, and a "low-bias" modulation scheme [81] for $\mathrm{MZ} \mathrm{LiNbO}_{3} \mathrm{EOMs}$, SFDRs of $\geq 120$ $\mathrm{dB} . \mathrm{Hz}^{2 / 3}$ had also been reported for these links.

Fig. 21 shows the SFDR measured for a homodyne coherent-AM link in which a $\mathrm{MZ} \mathrm{LiNbO}_{3}$ EOM (with a half-wave voltage $\mathrm{V}_{\pi}$ of $\sim 5.4 \mathrm{~V}$ at $9 \mathrm{GHz}$ ) was biased at its "carrier-suppression" point for efficient modulation of the optical field [82]. 


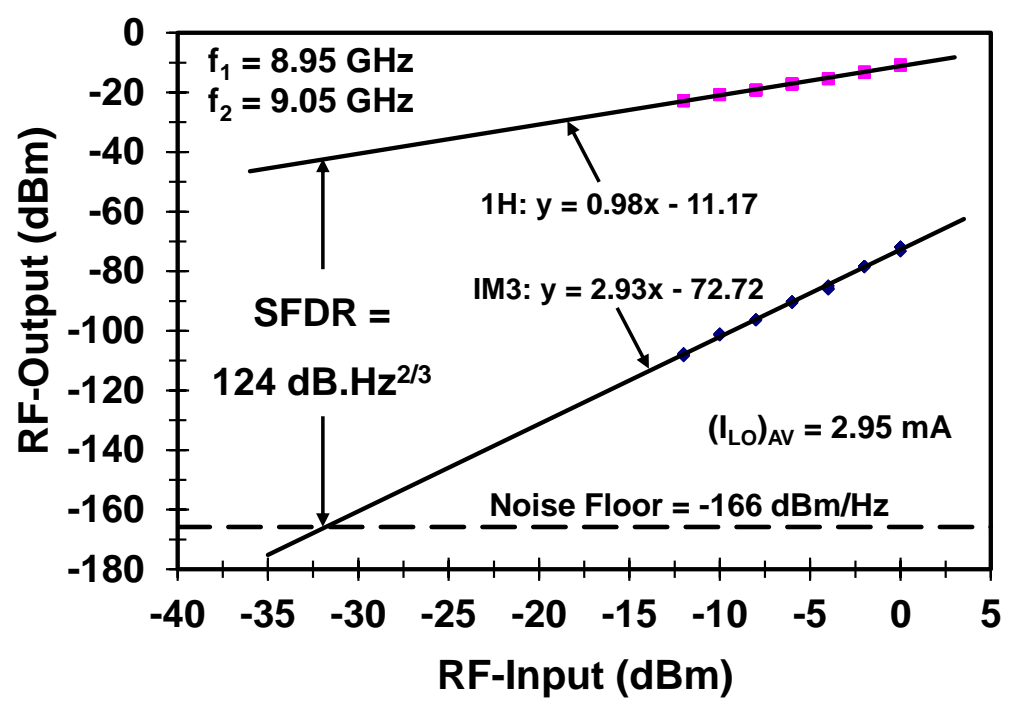

Fig.21 Measured two-tone SFDR of a Coherent AM Link that operated at $9 \mathrm{GHz}$. In the figure, $1 \mathrm{H}=1^{\text {st }}$ harmonic, $I M 3=3^{\text {rd }}$ order intermodulation product $\left(2 f_{i}-f_{j}, i=1,2\right)$ measured from the link receiver output.

A $\lambda_{\circ} \sim 1550 \mathrm{~nm}$ master-oscillator power-amplifier (MOPA) source was used to generate the optical input for the EOM. Before feeding the MOPA, part of the single-frequency laser-output (the master-oscillator) was tapped off to form an $\mathrm{LO}$ for our coherent receiver, which was comprised of a directional coupler and a pair of double-balanced PIN photodetectors. As shown, we achieved a SFDR of $124 \mathrm{~dB} . \mathrm{Hz}^{2 / 3}$, using photodiode $\mathrm{I}_{\mathrm{dc}}$ that was only $\sim 3 \mathrm{~mA}$. Therefore, the above carrier-suppressed coherent-AM link is an alternative approach that allows us to bypass the use of high power photodiodes at the link receiver. The low $I_{d c}$ used was also responsible for the relatively low shot-noise limited noise-floor of $-166 \mathrm{dBm} / \mathrm{Hz}$ measured. Unlike the "lowbias" approach, the coherent AM approach does not have a single-octave bandwidth restriction due to the occurrence of second harmonics $(2 \mathrm{H})$ in the MZ EOM. However, compared with recent link demonstrations [79-80], the gain of the above coherent AM link was still low. Since the link gain $\mathrm{G}$ was inversely proportional to $\mathrm{V}_{\pi}^{2}$, $\mathrm{G}$ can be enhanced by $10 \mathrm{~dB}$ if we use an EOM that has an RF- $\mathrm{V}_{\pi}$ of $\sim 1.7 \mathrm{~V}$ at $9 \mathrm{GHz}$. With the enhancement in $\mathrm{G}$, the NF of the above coherent AM link could be dropped to $\sim 9 \mathrm{~dB}$.

The performance (G, NF and SFDR) of our RF-photonic link provides the underpinning for the insertion of photonics technology in microwave systems. For example, the sensitivity of an antenna remoting link is directly related to its noise figure. Of key importance is the lowering of 
the $\mathrm{EOM} \mathrm{RF-V_{ \pi }}$. In addition, the highest frequency of our RF-input ( $\left.R F_{\text {in }}\right)$ for many of the applications described above is set by the modulator bandwidth. Since G is proportional to $\left(\mathrm{I}_{\mathrm{dc}} / \mathrm{V}_{\pi}\right)^{2}$, the use of a higher $\mathrm{I}_{\mathrm{dc}}$ in conjunction with high-linearity UTC photodiodes can help enhance the link gain. However, at long link lengths, the high optical power propagating down a fiber or waveguide could also generate undesirable nonlinear effect such as stimulated Brillouin scattering (SBS). For applications that require a frequency-bandwidth less than an octave, the "low-bias" link approach mentioned above will enable us to achieve a NF $<10 \mathrm{~dB}$ and SFDR > $122 \mathrm{~dB} . \mathrm{Hz}^{2 / 3}$. Finally, the digital linearization of photonic links $[10,48,67]$ that capitalize on the perfect linearity of optical phase modulation could also become a viable approach for enhancing the link SFDR.

\section{Discussion and Outlook}

In summary, we have reviewed several demonstrations in a few topical areas that are traditionally grouped under the technology "microwave photonics (MWP)". They include: the Optical Steering of Microwave Phased Arrays, Photonic-assisted ADCs, and RF-Photonic filtering. While not intended to be comprehensive or exhaustive, our discussions were geared towards illustrating where photonics can make a difference for wideband antenna steering, A/D conversion and signal processing. Because of its high carrier frequency ( $f_{\text {opt }}$ ) and low-loss distribution capabilities, MWP will make a even more significant impact as we move towards higher microwave frequencies, e.g., beyond the Ku-band. Even at W-band, the ratio of the signal-input frequency to $\mathrm{f}_{\mathrm{opt}}$ is still less than $0.1 \%$. Applications such as fiber-radio and $\mathrm{THz}$ wireless communication are already exploring the use of high frequency photodetectors at Vand W-bands for signal generation $[15,83]$. To keep this paper to a reasonable length, we have not provided a Section that highlights photonic signal generation, e.g., via use of optoelectronic oscillators (OEO) [13]. OEOs have demonstrated the capability to generate widely tunable microwave signals that also possess exceptional spectral purity. A recent review of OEOs is given in Ref. 84.

The early work in MWP were aimed at demonstrating the modulation of high frequency RFsignals onto optical carriers, using photonic devices (directly modulated lasers or EOMs) that were still in their infancy. However, they provided opportunities [e.g., 17, 19-20] for workers in 
the optoelectronics field to interact with microwave/antenna engineers responsible for integrating the "photonic black boxes" into their host-systems. Based on these interactions, link models were developed to estimate the link G, NF and SFDR, parameters that became recognized as key metrics for system insertion [3, 12]. This phase of MWP development also coincided with the rapid growth of new "photonic tools" (e.g., EDFAs, WDM muxes/demuxes, fiber Bragg gratings) for telecommunication. Much of the MWP work described in this paper were accomplished by leveraging co-development in these technologies.

Although many of its attributes for enhancing the performance of microwave systems had been demonstrated, MWP subsystems/components still need to demonstrate the size, weight and power (SWAP) metrics that meet the expectations of the microwave/antenna system integrators. We envision that many of these metrics for SWAP, as well as requirements for phase-stability can be met with use of integrated form-factors for MWP components. This stage of MWP evolution - aptly called "Integrated MWP" - has already started with the development of process design kits (PDKs) and foundry processes for component fabrication. These developments could also lead to potential cost reduction for MWP components. Looking back, the push towards MWP integration is not entirely new. Soon after our demonstration of the dual-band transmit array [17], a monolithic TTD PIC [see Fig.22] was fabricated [85] - albeit with higher insertion loss - with the intention of replacing the hybrid modules reported in [17]. In subsequent years, more emphasis - mostly involving the hybrid integration of subsystems - was undertaken to improve the link metrics and demonstrate the "value" of adding photonics to microwave systems. The advancement of material growth and fabrication technologies during these intervening years should facilitate the present evolution towards integrated MWP. 


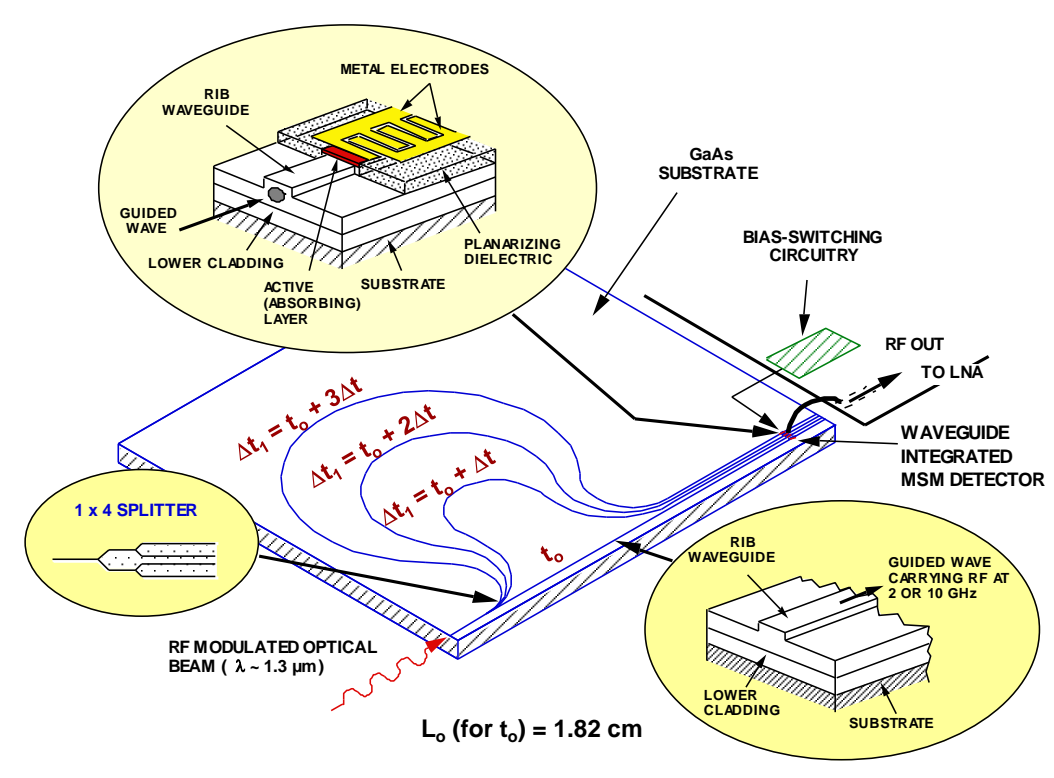

Fig.22 Monolithic GaAs PIC ( $2 \mathrm{~cm} \times 2 \mathrm{~cm})$ fabricated to steer phased array operating from L- to X-band via use of detector-bias controlled delay-line switching [85]

However, unlike CMOS electronics, photonic subsystems are typically comprised of components fabricated with use of diverse material technologies (e.g., III-V, Si or $\mathrm{LiNbO}_{3}$ ). Therefore, heterogeneous integration technologies will be needed to form these PICS. With improved formfactors, MWP components will find more system insertion opportunities not only in defense systems, but also for commercial markets such as fiber radio and high frequency wireless communication.

\section{Acknowledgements}

The author would like to acknowledge his collaborators at the HRL Laboratories, Malibu, CA. The true-time-delay beamforming work was a collaborative effort undertaken by the HRL Laboratories and several business units of the legacy Hughes Aircraft Company, parts of which had since been acquired by The Boeing Company and Raytheon. The work reported in this paper was partially funded by DARPA and the Air Force Research Laboratories (AFRL) over multiple contract awards. 


\section{References:}

1. H. Zmuda, and E. N. Toughlian (Ed.), Photonic Aspects of Modern Radar (Artech, 1995)

2. W.S.C. Chang (Ed.), RF Photonic Technology in Optical Fiber Links, Cambridge University Press (2002).

3. C. H. Cox III, Analog Optical Links: Theory and Practice (Cambridge Univ. Press, Cambridge, U.K., 2004).

4. A. Seeds and K. Williams, "Microwave Photonics," J. Lightw. Technol., Vol. 24, No. 12, pp.4628-4641, 2006.

5. J. Yao, "Microwave Photonics", J. Lightw. Technol., Vol. 27, No.3, pp.314-335, 2009.

6. S. A. Pappert, R. Esman, B. Krantz, "Photonics for RF Systems", Paper TuA3, IEEE Avionics, Fiber-Optics and Photonics Technology Conference, 2008.

7. Capmany, J. and Novak, "Microwave Photonics combines two worlds", Nature Photon. 1, pp.319-330 (2007)

8. R. Williamson and R. Esman, "RF Photonics", J. Lightw. Technol., Vol. 26, No. 9, pp. $1145-1153,2008$

9. See C. Lee (Ed.), Microwave Photonics (Second Edition), CRC Press (2013) and references cited therein.

10. T. Clark and R. Waterhouse, "Photonics for RF Front Ends", IEEE Microwave Magazine, Vol.12, No.3, 87-95, 2011

11. See D. Marpaung, C. Roeloffzen, R. Heiderman, A. Leinse, S. Sales and J. Capmany, "Integrated Microwave Photonics," Laser and Photonics Review, Nov.20, 2012 and references cited therein.

12. See V. Urick, J. McKinney, K. J. Williams, Fundamentals of Microwave Photonics, Wiley (2015) and references cited there in.

13. X.S. Yao, L. Maleki, and D. Eliyahu, "Progress in the Opto-Electronic Oscillator - A Ten Year Anniversary Review," Microwave Symposium Digest, 2004 MTT-S, International, pp.287-290

14. J. Yao, F. Zeng, "Photonic Generation of Ultrawideband Signals," J. Lightw. Technol., Vol. 25, No.11, pp.3219-3235, 2007

15. S. Ben Yoo, R. Scott, D. Geisler, N. Fontaine, F. Soares, "Terahertz Information and Signal Processing by RF-Photonics," IEEE Transactions on Terahertz Science and Technology, Vo.2, No.2, pp.167-176, 2012.

16. J. Capmany, J. Mora, I. Gasulla, J. Sancho, J. Lloret and S. Sales, "Microwave Photonic Signal Processing," J. Lightw. Technol., Vol. 31, no.4, pp.571-586, 2013 
17. W. Ng, A. A.Walston, G. L. Tangonan, J. J. Lee, I. L. Newberg, and N. Bernstein, “The first demonstration of an optically steered microwave phased array antenna using truetime-delay," J. Lightw. Technol., Vol. 9, no. 9, pp. 1124-1131, 1991.

18. See M. Frankel, P. Matthews, R. Esman and L. Goldberg, "Practical optical beamforming networks", Optical and Quantum Electronics, 30(11-12), 1033-1050, 1998 and references cited therein.

19. See Chapter 12 of Ref. 2 and also W. Ng, R. Loo, G. Tangonan, J.J. Lee, S. Livingston and F. Rupp, "Photonically Controlled Phased Arrays", SPIE Proceedings Vol. 3463, Photonics and Radio Frequency II, pp.8-17, 1998, and references cited therein.

20. R. Loo, G. Tangonan, H.W. Yen, V. Jones, W. Ng, J. Lewis, J.J. Lee and S. Livingston, "Photonics for Phased Array Antennas," SPIE Proceedings 2844, pp. 234-240, 1996

21. T. Kominato, Y. Hida, M. Itoh, H. Takahashi, S. Sohma, T. Kitoh and Y. Hibino, "Extremely low-loss $(0.3 \mathrm{~dB} / \mathrm{m})$ and long silica-based waveguides with large width and clothoid curve connection," in Proceedings of ECOC, Paper TuI 4.3, (Stockholm, Sweden, 2004)

22. J. Bauters, M. Heck, D. John, J. Barton, C. Bruinink, A. Leinse, R. Heideman, D. Blumenthal, J. Bowers, "Planar waveguides with less than $0.1 \mathrm{~dB} / \mathrm{m}$ propagation loss fabricated with wafer bonding," Opt. Express 19(24), 24090-24101 (2011)

23. K. Takada, H. Yamada, Y. Hida, Y. Ohmori and S. Mitachi, "Rayeigh backscattering measurement of $10 \mathrm{~m}$ long silica-based waveguides", Electron. Lett., 1996, 32 (18), pp. 1665-1667.

24. Y. Hida, and Y. Hibino, "Ultra-high density AWGs composed of super-high $\triangle$ PLCs", Paper ThC6, the Optical Fiber Conference (OFC) 2002, March 17-22, Anaheim, CA

25. J. Corral, J. Marti, J. Fuster and R. Laming, "True Time-Delay Scheme for Feeding Optically Controlled Phased-Array Antennas Using Chirped-Fiber Gratings," IEEE Photon. Technol. Lett., Vol. 9, No.11, pp.1529-1531, 1997.

26. Y. Liu, J. Yao and J. Yang, "Wideband true-time-delay beam former that employs a tunable chirped fiber grating prism," Appl. Opt., Vol. 42, No.13, pp.2273-2277, 2003

27. S. Yegnanarayanan, P. Trinh and B. Jalali, "Recirculating photonic filter: a wavelengthselective time delay for phased-array antennas and wavelength code-division multiple access," Optics Lett., Vol. 21 (10), pp.740-742, 1996.

28. See C. Doerr and K. Okamoto, “Advances in Silica Planar Lightwave Circuits,” J. Lightw. Technol., Vol. 24, no.12, pp. 4763-4789, 2006 and references cited therein.

29. See for example, A. Ward, D. Robbins, G. Busico, E. Barton, L. Ponnampalam, J. Duck, N. Whitbread, P. Williams, D. Reid, A Carter and M. Wale, "Widely tunable DS-DBR Laser with Monolithically Integrated SOA: Design and Performance”, IEEE J. Quantum Electron., vol. 11 (1), pp.149-156, 2005 and reference cited therein. 
30. J. Simsarian, M. Larson, H. Garrett, H. Xu and T. Strand, "Less than 5-ns Wavelength Switching With an SG-DBR Laser", IEEE Photon. Technol. Lett., Vol. 18, No.4, pp.565$567,2006$.

31. A. Meijerink et al, "Novel Ring Resonator-Based Integrated Photonic Beamformer for Broadband Phased Array Receive Antennas-Part I: Design and Performance Analysis", J. Lightw. Technol., Vol. 28, no.1, pp. 3-18, 2010

32. L. Zhuang et al, "Novel Ring Resonator-Based Integrated Photonic Beamformer for Broadband Phased Array Receive Antennas-Part I: Design and Performance Analysis", J. Lightw. Technol., Vol. 28, no.1, pp. 19-31, 2010

33. M. Burla et al, "Multiwavelength-Integrated Optical Beamformer Based on Wavelength Division Multiplexing for 2-D Phased Array Antennas,” J. Lightw. Technol., Vol. 32, no.20, pp. 3509-3520, 2014

34. P. Morton and J. Khurgin, "Microwave Photonic Delay Line with Separate Tuning of the Optical Carrier,” IEEE Photon. Technol. Lett., Vol. 21, No.22, pp.1686-1688, 2009

35. C. Roeloffzen et al, "Integrated Optical Beamformers," Paper Tu3F.4, the 2015 Optical Fiber Conference (OFC), Los Angeles, CA, March 22-26, 2015.

36. C. Cox, E. Ackerman, G. Betts, J. Prince, "Limits on the Performance of RF-over-Fiber Links and Their Impact on Device Design," IEEE Trans. Microw. Theory Tech. 54 (2), 906-920, Feb., 2006

37. E. Ackerman, W. Burns, G. Betts, J. Chen, J. Prince, M. Regan, H. Roussell and C. Cox, "RF-Over-Fiber Links With Very Low Noise Figure", J. Lightw. Technol., Vol. 26, no. 15, pp. 2441-2448, 2014

38. R. H. Walden, “Analog-to-digital converter survey and analysis”, IEEE J. Sel. Areas Communica., 17(4): 539-550, 1999.

39. R. H. Walden, “Analog-to-Digital Conversion in the Early Twenty-first Century," Wiley Encyclopedia of Computer Science and Engineering, B. Wah, Ed. (Wiley), 2008.

40. W. Ng, Y. M. So, R. Stephens and D. Persechini, "Characterization of the jitter in a mode-locked Er-fiber laser and its application in photonic sampling for analog-to-digital conversion at 10 Gsamples/s", J. Lightw. Technol., Vol.22, No.8, pp.1953-1961 (2004)

41. W. Ng, Y. So, "Characterisations of absolute phase noise in fibre-laser modelocked by sapphire-loaded cavity resonator oscillator at $10 \mathrm{GHz}$," Electron. Lett., vol. 40, no.11, pp. 672-674, 2004

42. P. Juodawlkis et al, "Optically Sampled Analog-to-Digital Converters,” IEEE Trans. Microw. Theory Tech. 49 (10), 1840-53 2001.

43. See F. Quinlan, S. Ozharar, S. Gee and P J Delfyett, "Harmonically mode-locked semiconductor-based lasers as high repetition rate ultralow noise pulse train and optical frequency comb sources," J. Opt. A: Pure Appl. Opt. 11 (2009), pp.1-23 and references cited there in. 
44. D. Esman, A. Wiberg, N. Alic and S. Radic, "High Resolution Broadband Photonic Sampled ADC: 8.0 ENOB at 40 GHz", OECC/ACOFT 2014, Melbourne, Australia, 6-10 July 2014.

45. L. Luh, W. Ng, J. F. Jensen, D. Le, D. Persechini, S. Thomas, C. Fields and J. Lin, "A 10.24GSPS Photonic Sampled Bandpass $\Delta \Sigma$ Modulator Direct-Sampling at $12 \mathrm{GHz}$ ", Proceedings of the IEEE 2005 Custom Integrated Circuits Conference, pp.387-390.

46. See Fig.12.6, Section 12.3 of Ref.2.

47. A. Fard, S. Gupta, and B. Jalali, "Photonic time-stretch digitizer and its extension to realtime spectroscopy and imaging," Laser Photonics Rev., 1-57 (2013)

48. W. Ng, T. D. Rockwood, G. A. Sefler, G. C. Valley, "Demonstration of a Large StretchRatio (M=41) Photonic Analog-to-Digital Converter With 8 ENOB for an Input Signal Bandwidth of 10 GHz," IEEE Photon. Technol. Lett., Vol. 24, no.14, pp.1185-1187, 2012

49. G. Sefler, J. Chou, J. Conway, G. C. Valley, "Distortion correction in a high-resolution time-stretch ADC scalable to continuous time", J. Lightwa. Technol., Vol. 28, No. 10, pp. 1468-1476, (2010).

50. G. Sefler and G. C. Valley, "Mitigation of Group-Delay-Ripple Distortions for Use of Chirped Fiber-Bragg Gratings in Photonic Time-Stretch ADCs", J. Lightwa. Technol., Vol. 31, No. 7, pp. 1093-1100, (2013)

51. W. Ng, T. Rockwood, A. Reamon, "Demonstration of Channel-Stitched Photonic TimeStretch Analog-to-Digital Converter with ENOB $\geq 8$ for a $10 \mathrm{GHz}$ Signal Bandwidth", Abstract of Paper 26.2 in the Program of the GOMACTech-14 Conference, Charlston, SC, March 31- April 3, 2014.

52. R. Minasian, "Photonic Signal Processing Microwave Signals", IEEE Trans. Microw. Theory Tech. 54 (2), 832-846, 2006

53. J. Capmany, B. Ortega, D. Pastor, S. Sales, "Discrete-Time Optical Processing of Microwave Signals”, J. Lightw. Technol., Vol. 23, No.2, pp.314-335, 2005.

54. J. Capmany, B. Ortega and D. Pastor, “A Tutorial on Microwave Photonic Filters,” J. Lightw. Technol., Vol. 24, No.1, pp.201-229, 2006

55. E. Hamidi, D. Leaird and A. Weiner, "Tunable Programmable Microwave Photonic Filters Based on an Optical Frequency Comb", IEEE Trans. Microw. Theory Tech. 58 (11), 3269-3278, 2010.

56. V. Supradeepa, C. Long, R. Wu, F. Ferdous, E. Hamidi, D. Leaird and A. Weiner, "Comb-based radiofrequency photonic filters with rapid tenability and high selectivity," Nature Photon. 6, pp.186-194 (2012)

57. W. Ng, D. Persechini and S. Bourgholtzer, "Bipolar transversal filters with large sidelobe suppression implemented using WDM technologies," Electron. Lett., Vol. 44 (5), pp.372$373,2008$. 
58. M. Rasras et al, "Demonstration of a Fourth-Order Pole-Zero Optical Filter Integrated Using CMOS Processes”, J. of Lightwave Technol., Vol.25, No.1, Jan. 2007.

59. P. Dong, N. Feng, D. Feng, W. Qian, H. Liang, D. Lee, B. Luff, B. Banwell, A. Agarwal, P. Toliver, R. Menendez, T. Woodward and M. Asghari, "GHz-bandwidth optical filters based on high-order silicon ring resonators”, Opt. Express 18 (23), 23784-23789 (2010)

60. E. Norberg, R. Guzzon, J. Parker, L. Johansson and L. Coldren, "Programmable Photonic Microwave Filters Monolithically Integrated in InP-InGaAsP," J. of Lightwave Technol., Vol. 29, No.11, June, 2011

61. P. Orlandi, F. Morichetti, M. Strain, M. Sorel, P. Bassi and A. Melloni, "Photonic Integrated Filter with Widely Tunable Bandwidth,” J. of Lightwave Technol., Vol. 32, No.5, p.897-907, March, 2014

62. K. Jinguji, "Synthesis of Coherent Two-Port Optical Delay-Line Circuit with Ring Waveguides”, J. of Lightwave Technol., Vol.14, pp.1882-1898, Aug. 1996

63. See C. K. Madsen and J. H. Zhao, "Optical Filter Design and Analysis, A Signal Processing Approach," $1^{\text {st }}$ ed., New York: Wiley, 1999, Ch.6, and references cited therein.

64. R. Nagarajan, et al, "InP Photonic Integrated Circuits,” IEEE J. Sel. Topics Quantum Electron., vol.16, no.5, pp.1113-1125, 2010.

65. J. McKinney, M. Godinez, V. Urick, S. Thaniyavarn, W. Charczenko, and K. Williams, "Sub-10-dB Noise Figure in a Multiple-GHz Analog Optical Link," IEEE Photon. Technol. Lett., Vol. 19, no.7, pp.465-467, 2007.

66. J. Shin and N. Dagli, "Ultralow Drive Voltage Substrate Remove GaAs/AlGaAs ElectroOptic Modulators at 1550 nm", IEEE J. Select. Topics Quantum Electron., Vol. 19, no.6, 2013.

67. See, for example, A. Agarwal, T. Banwell and T. K. Woodward, "Optically filtered microwave photonic links for RF signal processing applications," J. Lightw. Technol., vol.29, no.16, pp.2394-2401, 2011.

68. W. Ng, "RF-Photonic Transversal Filter Method and Apparatus," U.S. Patent 8,121,485 B2, issued 2/21/2012

69. See, for example, A. Yariv, "Universal relations for coupling of optical power between microresonators and dielectric waveguides", Electronics Letters, Vol.36, No., pp.321-322 (2000).

70. W. Ng, T. Rockwood, D. Persechini and D. Chang, "High-Q Si microresonators formed by substrate transfer on silica waveguide wafers", Optics Express 18(26), 27004-27015 (2010) 
71. H. Rokhsari and K. J. Vahala, "Ultralow Loss, High Q, Four Port Resonant Couplers for Quantum Optics and Photonics”, Phys. Rev. Lett. 92 (25), 253905 1-4, 2004.

72. In the experiment of Fig.18, the silica MR was optically coupled with a pair of curved fiber-tapers. The intrinsic- $\mathrm{Q}\left(\mathrm{Q}_{\mathrm{o}}\right)$ was estimated as follows. By removing the drop-fiber, i.e., using only the incident fiber-taper, we measured the loaded-Q $\left(\mathrm{Q}_{\mathrm{cr}}\right)$ of the $\mathrm{MR}$ at critical coupling, when the throughput $\mathrm{E}_{\mathrm{e}}$ (see Fig.17b) was nulled. As the models of [69-70] show, we can then estimate the intrinsic- $Q, Q_{o}$, as: $Q_{o}=2 Q_{c r}$.

73. W. Ng, B. Limketkai, R. Hayes, D. Yap and P. Petre, "Reconfigurable Optical Filters formed by Integration of Electrically Tunable Microresonators", U.S. Patent $8,693,817 \mathrm{~B} 1$, issued 4/8/2014

74. B. Limketkai, W. Ng, T. Rockwood, “Optimization-based Synthesis of Photonic IIR filters Accounting for Internal Losses in Microresonators," to be submitted for publication in IEEE Trans. Circuits Syst. I.

75. See, for example, L. Zhuang, D. Marpaung, M. Burla, W. Beeker, A. Leinse and C. Roeloffzen, "Low-loss, high-index-contrast $\mathrm{Si}_{3} \mathrm{~N}_{4} / \mathrm{SiO}_{2}$ optical waveguides for optical delay lines in microwave photonics signal processing," Opt. Express, 19(23), 2316223170, and references cited therein.

76. Z. Wang, S. Chang, C. Ni and Y. Chen, “A High-Performance Ultracompact Optical Interleaver Based on Double-Ring Assisted Mach-Zehnder Interferometer", IEEE Photon. Technol. Lett., Vol. 19, No.14, pp.1072-1074, 2007

77. K. Williams, D. Tulchinsky and J. Campbell, "High Power Photodiodes", Proceedings of the 2007 IEEE International Topical Meeting on Microwave Photonics, pp. 9-13

78. Rouvalis, et al, "High-Power and High-Linearity Photodetector Modules for Microwave Photonic Applications,” J. of Lightwave Technol., Vol. 32, No.20, pp.3810-3816, Oct. 2014.

79. Urick, et al "Long-Haul Analog Photonics," J. of Lightwave Technol., Vol. 29, No.8, pp.1182-1204, April, 2011.

80. X. Xie, Q. Zhou, A. Beling and J. Campbell, "High-Gain, Low-Noise-Figure, and HighLinearity Analog Photonic Link Based on a High-Performance Photodetector,” J. of Lightwave Technol., Vol. 32, No.21, pp.4187-4192, Nov., 2014. 
81. A. Karim and J. Devenport, "Optimization of Linearity Figure of Merit for Microwave Photonic Links,” IEEE Photon. Technol. Lett., Vol. 21, No.13, pp.950-952, 2009

82. W. Ng, "Coherent Technologies for Analog Transmission with Enhanced Linearity," Frontiers in Optics (FiO) Invited Paper (FMJ1), 2006 OSA Annual Meeting.

83. A. J. Seeds et al, “Coherent terahertz photonics," Optics Express 21(19), 22988-23000 (2013)

84. L. Maleki, “The Opto-Electronic Oscillator (OEO): Review and Recent Progress”, European Frequency and Time Forum (EFTF), 2012, p.497-500.

85. W. Ng, D. Yap, A. Narayanan, A. Walston, "High-Precision Detector-Switched Monolithic GaAs Time-Delay Network for the Optical Control of Phased Arrays", IEEE Photon. Technol. Lett., Vol. 6, no.2, pp.231-234, 1994. 


\section{Figure Captions:}

Fig.1 a. Photograph of the EHF transmit array [17] in which the L- and X-band antennas were designed to have the same array length $\mathrm{L}$ b. Squintless beams formed at $1.9 \mathrm{GHz}(\mathrm{L}-$ band) and 9.0 GHz (X-band) for $\theta_{0}=-24^{\circ}$ c. Measured angle vs programmed angle of antenna beam formed at $9 \mathrm{GHz}$

Fig.2 a. Photograph of the L-band conformal radar [19-20] with a $10 \mathrm{ft}$ radius of curvature b. Transmit beam pattern obtained for $\mathrm{a}+30^{\circ}$ scan $\mathrm{c}$. Receive beam pattern obtained for a $60^{\circ}$ scan

Fig.3 The generation of stepped time-delays from an integrated chip comprised of WG delay-loops and AWG mux/demux via the switching of optical wavelengths $\lambda_{1} . . \lambda_{\mathrm{N}}$. In the figure, PD denotes photodetector.

Fig.4 a. Cascaded optical ring resonator (ORR) integrated on a chip to accomplish tunable delay over a wider optical bandwidth b. Time-delay obtained for cascaded ORR 1 and ORR 2, showing the tradeoff between bandwidth and ripple [35]

Fig.5 a. Experimental setup for measuring the absolute $\phi$-noise of a fiber laser ML by a SLCR oscillator at the frequency of $f_{M L}=10.24 \mathrm{GHz}$ b. Measured single sideband $\phi$-noise L(f) of SLCR oscillator and the ML fiber laser.

Fig.6 Photonic A/D conversion using low jitter ML pulses for impulse sampling and InPHBT $\triangle \Sigma$ ADC for quantization. The analog-input was impressed as an envelope on the pulse train via use of an EOM. The reference synthesizer used in Ref. 45 was a SLCR $10.24 \mathrm{GHz}$ oscillator

Fig.7 InP-HBT $\Delta \Sigma$ quantizer optoelectronic integrated circuit (OEIC) with integrated photodetectors, analog circuit (analog loop-filter and integrator), and a comparator (1-bit quantizer) [45]

Fig.8 Measured SNR vs the relative input-power (at $f_{i n}=12 \mathrm{GHz}$ ) to the EOM. The inset shows the $1.76 \mathrm{GHz}$ downconverted signal located at the quantization-notch of the InP-HBT $\Delta \Sigma \mathrm{ADC}$ 
Fig.9 Schematic of a time-stretched (TS) photonic ADC system. The Filter/Time-Delay Module after $\mathrm{D}_{1}$ provides an edge-overlap of the SC pulses (formed by spectral slicing with filters in the module) for Ch-stitching during post processing

Fig.10 Schematic of the M=41 TS photonic ADC system [48] formed using an actively ML $\mathrm{SC}$ laser source with pulse repetition period of $\mathrm{T}_{\mathrm{m}}=8 \mathrm{nsec}$. In the figure, $\mathrm{PC}$ and $\Delta \lambda$ denote, respectively, polarization controller and $\mathrm{BW}$ of the filters $\left(\mathrm{H}_{1}, \mathrm{H}_{2}\right)$. A real time $(\mathrm{RT})$ digitzer that had an analog bandwidth of $16 \mathrm{GHz}$ was used to take the data of Fig.11.

Fig.11 a. RF-Modulation extracted from the arc-sine algorithm for a $10 \mathrm{GHz}$ analog-input stretched to $\mathrm{f}_{\mathrm{TS}}=242.3 \mathrm{MHz}$, and its sine-fit used for ENOB evaluation [48] b. ENOB (lower trace, with a mean value of 4.38) obtained for a $16 \mathrm{GHz}$ noise-bandwidth of the RT digitizer, and the ENOB (upper trace, with a mean value of 8.27) obtained after digital filtering with a lowpass-filter designed to have a cutoff at $\mathrm{f}_{\mathrm{TS}}$.

Fig.12 Plot of the transfer function $\operatorname{HDIP}\left(f_{\text {in }}\right)$ [Eq. 4] modeling the dispersion induced penalty (DIP) in a TS photonic ADC system

Fig.13 RF-photonic filters in an aperture-remoted microwave antenna system. After filtering, the filter-output that remains in the optical domain is distributed to multiple physical locations for photodetection, high resolution A/D conversion, and DSP

Fig.14 Schematic of bipolar FIR RF-photonic filter implemented using WDM technologies, a length of chirped fiber grating (CFG) and a differential photoreceiver [57].

Fig.15 a. Optical spectrum of a WDM comb windowed to form a 20-tap Dolph-Chebyshev filter. b. RF response of the FIR filter formed with use of the tap-apodization shown in Fig.15a. In the figure, $\mathrm{f}_{\mathrm{c}}$ and $\Delta \mathrm{f}$ denote, respectively, the center frequency of passband and its $-1 \mathrm{~dB}$ PBW.

Fig.16 Reconfiguration of the filter-passband from a 30-tap unipolarity ( $\lambda$-reused) FIR filter to a 20-tap bipolar filter. Both filters were formed by adopting Dolph-Chebyshev coefficients for tap-apodization. 
Fig.17 a. Fabry-Perot (FP) etalon b. Microresonator (MR) evanescently coupled to incident and drop waveguides c. Digital filter model of the drop-output for the MR of Fig.17b

Fig.18 Measured throughput (Throu.) and Drop-output (Drop) of a silica/Si MR with an intrinsic- $\mathrm{Q}\left(\mathrm{Q}_{\mathrm{o}}\right)$ of $\mathrm{Q}_{\mathrm{o}}=2.56 \times 10^{7}$. The evanescent coupling to the MR was accomplished with use of fiber-tapers.

Fig.19 Second-order unit cell formed by coupling microresonators $M R_{1}$ and $M R_{2}$ to an input and an output directional coupler. A phase-shifter $\delta$ provides tuning of the relative phase between the two arms.

Fig.20 Bandpass filter formed by cascading two of the unit cell shown in Fig.19: a. Center $f_{c}$ of passband tuned to $3 \mathrm{GHz}$ b. Center $\mathrm{f}_{\mathrm{c}}$ of passband tuned to $8 \mathrm{GHz}$

Fig.21 Measured two-tone SFDR of a Coherent AM Link that operated at $9 \mathrm{GHz}$. In the

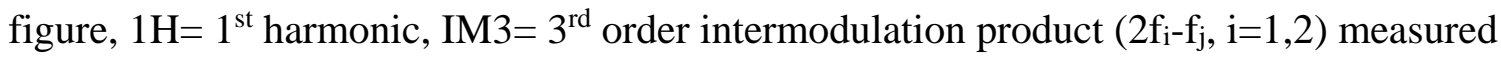
from the link receiver output

Fig.22 Monolithic GaAs PIC (2 cm x $2 \mathrm{~cm})$ fabricated to steer phased array operating from L- to X-band via use of detector-bias controlled delay-line switching [85] 